\author{
Universidade de São Paulo \\ Instituto de Física
}

\title{
Matéria de Quarks Fria sob Campo Magnético Forte
}

\author{
Theo Ferraz Motta
}

Orientador: Prof. Dr. Fernando Silveira Navarra

Dissertação mestrado apresentada ao Instituto de

Física para a obtenção do título de Mestre em Ciências

Banca Examinadora:

Prof. Dr. Fernando Silveira Navarra (USP)

Profa. Dra. Laura Paulucci Marinho (UFABC)

Prof. Dr. Germán Lugones (UFABC) 


\section{FICHA CATALOGRÁFICA \\ Preparada pelo Serviço de Biblioteca e Informação do Instituto de Física da Universidade de São Paulo}

Motta, Theo Ferraz

Matéria de quarks fria sob campo magnético forte. São Paulo, 2017.

Dissertação (Mestrado) - Universidade de São Paulo. Instituto de Física. Depto. de Física Experimental.

Orientador: Prof. Dr. Fernando Silveira Navarra

Área de Concentração: Física

Unitermos: 1. Cromodinâmica quântica; 2.; Estrelas de nêutrons;

3. Campo magnético.

USP/IF/SBI-035/2017 


\author{
Universidade de São Paulo \\ Instituto de Física
}

\title{
Cold Quark Matter under Strong Magnetic Field
}

\author{
Theo Ferraz Motta
}

Advisor: Prof. Dr. Fernando Silveira Navarra

Masters Disstertation submitted to the Institute of Physics in fulfillment of the requirements

for the degree of Master of Sciences

Examination Committee:

Prof. Dr. Fernando Silveira Navarra (USP)

Profa. Dra. Laura Paulucci Marinho (UFABC)

Prof. Dr. Germán Lugones (UFABC) 



\title{
Resumo
}

Esta dissertação apresenta uma breve revisão introdutória de alguns aspectos importantes de astrofísica nuclear e da fenomenologia do plasma de quarks e glúons a baixas temperaturas. Acredita-se que tal estado da matéria existe no núcleo de estrelas de nêutron e possivelmente de outros objetos compactos em astrofísica. Uma equação de estado para tal sistema é derivada incluindo as influências dos condensados de glúon e do campo magnético que também é uma característica importante das estrelas de nêutron. Finalmente, essa equação de estado é aplicada para o estudo de estrutura estelar de estrelas compactas e alguns resultados importantes, publicados em [1], são discutidos.

Palavras-Chave: Estrela de Neutron, Cromodinâmica Quântica, Campo Magnético

\begin{abstract}
This dissertation presents a brief introductory overview of some key aspects of nuclear astrophysics and of the phenomenology of the quark gluon plasma at cold temperatures which is believed to exist inside the core of neutron stars and possible other compact astrophysical objects. An equation of state for this state of matter is derived incluing the influence of gluon condensates and the magnetic field which is also an important characteristic of neutron stars. And finally this equation of state is applied to the study of compact stellar structure and some important results, published in [1], are discussed.
\end{abstract}

Keywords: Neutron Star, Quantum Chromodynmics, Magnetic Field 



\section{Acknowledgements}

I would like to thank my family for all the support they gave me during my Masters, specially my mother Thais, my stepfather Milton, and my grandfather Ailton. I also would like to thank all of my dear friends that acompained me during this whole time, specially Rafaela whose support I can not thank enough. Fernando, my Professor Advisor, who encouraged me to pursue this project. David, for all the help and discussions. And CAPES for financial support. 

"Audentes fortuna iuvat" 



\section{Contents}

$\begin{array}{lll}\text { Abstract } & \text { v }\end{array}$

Acknowledgements vii

$\begin{array}{llr}1 & \text { Introduction } & \mathbf{1}\end{array}$

1.1 Cold Quark Matter . . . . . . . . . . . . . . . . . . . . . 2

1.2 Neutron Stars . . . . . . . . . . . . . . . 5

Stelar Evolution . . . . . . . . . . . . . 5

1.3 Properties of Neutron Stars . . . . . . . . . . . . . . . . 6

1.3.1 Equilibrium \& Stability . . . . . . . . . . 7

1.3.2 Mass, Radius . . . . . . . . . . . . . . . 8

1.3.3 Magnetic Field \& Rotation . . . . . . . . . . . . . . 10

1.3.4 Stellar Structure . . . . . . . . . . . . . . . . . . . . 10

1.4 Mean Field EOS with Magnetic Field . . . . . . . . . . . . . . . 11

2 Effective Lagrangian and Equation of State 15

2.1 QCD ............................ 15

2.2 Gluon Field Decomposition . . . . . . . . . . . . . . . . . . . . . . . . . . . 17

2.3 Gluon Condensates . . . . . . . . . . . . . . . . . . . 17

2.3.1 Properties of the Condensates . . . . . . . . . . . . 18

2.4 Effective Lagrangian . . . . . . . . . . . . . . . . . . . . . 19

2.5 Effective Lagrangian with Magnetic Field . . . . . . . . . . . 23

2.5.1 Equations of Motion . . . . . . . . . . . . . 25

2.5 .2 Landau Levels . . . . . . . . . . . . . . . . . . 25

Exact Solution . . . . . . . . . . . . . . . . 25

Quarks ..................... 27

2.6 Equation of State . . . . . . . . . . . . . . . 28

Energy, Entropy and Pressure . . . . . . . . . . . . 29

Zero Temperature Limit . . . . . . . . . . . . . . 33 
xii

3 Stellar Structure with a Magnetic Equation Of State 35

3.1 Stability Conditions . . . . . . . . . . . . . . . 37

Stability Window . . . . . . . . . . . . . 39

3.2 Mass-Radius Results . . . . . . . . . . . . . . . . . 41

$\begin{array}{lll}4 \text { Conclusion } & 47\end{array}$

$\begin{array}{ll}\text { A Derivation of the TOV equation } & 49\end{array}$

B Finite Temperature Fermion Field 53

$\begin{array}{ll}\text { Bibliography } & 65\end{array}$ 


\section{List of Figures}

1.1 QCD phase diagram $[3] \ldots \ldots \ldots \ldots \ldots$

1.2 Proton structure of two quarks "up" and one "down" . . . . . . 3

1.3 Illustration of Heavy Ion Collision. Image by Henning Weber / CERN . . . . . . . . . . . . . . . . . . 3

1.4 Deconfining quarks by increasing temperature or pressure . . 4

1.5 Pulsar illustration [5] . . . . . . . . . . . . . . . . 7

1.6 Measured NS masses [13] . . . . . . . . . . . . . . . . . . 12

1.7 Different EOSs predictions for the Mass $\times$ Radius diagram of neutron stars [9] . . . . . . . . . . . . . . . . . 13

1.8 Different types of neutron star [14]. INS: Isolated Neutron Star. MSP: Millisecond Pulsars. L(H)MXB: Low (High) mass x-ray binaries. CCO: Compact central objects. . . . . . . . . . 14

$1.9 P \times \dot{P}$ diagram [14] . . . . . . . . . . . . . . . . . 14

3.1 The splitting between the paralell and perpendicular pressures for $\mathrm{MQCD}$ and for the MIT bag model as a function of the magnetic field, where $\xi \doteq g_{h} / m_{G} \ldots \ldots \ldots 36$

3.2 Inner and Outer spherical limits . . . . . . . . . . . . 37

3.3 Stability windows defined by the conditions (1.3) to (1.4). . . 40

3.4 Stability diagram: baryon density ratio as function of the magnetic field. The points in the light gray area satisfy the conditions (1.3), (1.1) and (3.9). Points in the dark gray area satisfy also the condition (1.4). . . . . . . . . . . . . . . . 41

3.5 Mass-radius diagrams. Two values of the magnetic fields with $\mathcal{B}_{Q C D}$ and $\xi$ allowed by the stability conditions at central density $\rho_{B}=2.6 \rho_{0}$. The largest masses are 2.05 (mQCD) and 1.89 (MIT). In these cases $p_{\|}=p_{\perp}$ which permits the use

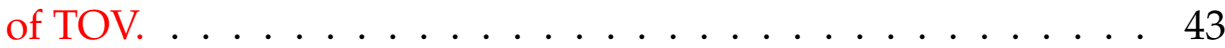


3.6 Mass-radius diagram for a fixed value of the magnetic field and baryon density with. (a) Fixing $\xi$ and varying $\mathcal{B}_{Q C D}$ : For $\mathcal{B}_{Q C D}=53 \mathrm{MeV} / \mathrm{fm}^{3}$ the higher masses are 2.22 and 2.04 (dotted lines). Whereas for $\mathcal{B}_{Q C D}=61 \mathrm{MeV} / \mathrm{fm}^{3}$ masses are of up to 2.06 and 1.93 (solid lines). (b) Fixing $\mathcal{B}_{Q C D}$ and varying $\xi$ : For $\xi=0.0015 \mathrm{MeV}^{-1}$ the largest masses are 2.20 and 2.06(dotted lines). Whereas for MIT $\left(\xi=0 \mathrm{MeV}^{-1}\right)$ the largest masses are 2.08 and 1.92 (solid lines). . . . . . . . . . . 44

3.7 Effects of the splitting of parallel and perpendicular pressures on the maximum masses calculated with the chemical potentials obeying the stability conditions (1.3), (1.1) and (3.9)), given by $\nu_{u}=300 \mathrm{MeV}, \nu_{d}=\nu_{s}=316.5 \mathrm{MeV}$ and $\mu_{e}=$ $16.5 \mathrm{MeV} \ldots \ldots \ldots \ldots \ldots \ldots \ldots$ 


\title{
List of Abbreviations
}

\author{
QCD Quantum Chromodynamics \\ mQCD mean-field Quantum Chromodynamics \\ QGP Quark-Gluon Plasma \\ SQM Strange Quark Matter \\ MFT Mean Field Theory \\ EOS Equation Of State \\ EM Electro-Magnetic \\ TOV Tolman-Oppenheimer-Volkof \\ GR General Relativity
}



Dedicated to Filomena, Bidau \& Lua 



\section{Chapter 1}

\section{Introduction}

Four forces are known to this day to exist governing the physical universe. Gravitational, Electromagnetic, Weak and Strong forces. Particularly the latter is one of the most puzzling. The strong force, ruled by the theory of Quantum Chromodynamics (QCD) tries to explain how quarks bind themselves together in hadrons such as protons and neutrons, how those hadrons behave and bind together to form a nucleus. It should also explain some other features of our universe such as the early stages of the evolution of our cosmos and other extreme states of matter formed at very high temperature or density [2].

QCD has already proven many times to be the fundamental theory of the strong interactions between particles. Its perturbative regime at high temperatures has been tested in heavy ion colliders such as LHC and RHIC. And with the help of Lattice simulations, QCD has given us many accurate predictions and results, such as the masses of hadrons.

However not all of the physical situations believed to be described by QCD, are well understood. The fact that quarks (fundamental constituents of QCD) at low energies are confined into colourless hadrons is yet to be mathematically proven. High density systems with low temperature such as those believed to be found inside compact stars, and even the lowest energy state of the theory (the QCD Vacuum) are yet to be completely understood.

In figure 1.1 we have what is believed to be the QCD phase diagram. Although many of those regions are not fully understood, with some theoretical assumptions we can perform calculations and predictions for experiments. 


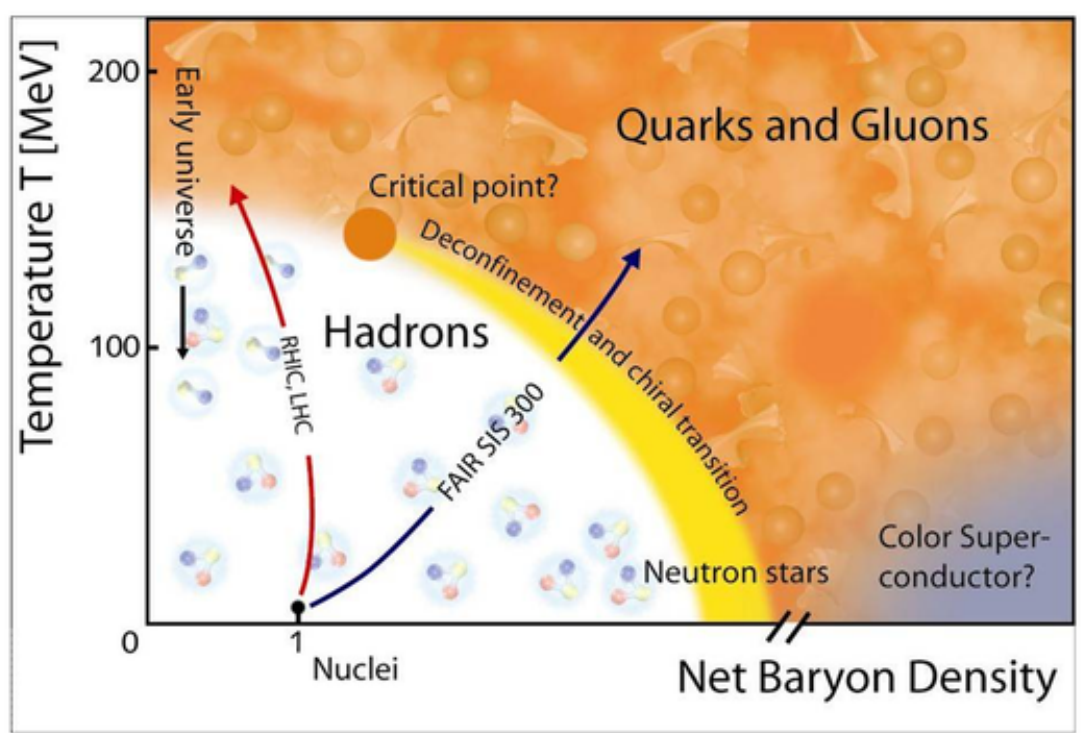

FIGURE 1.1: QCD phase diagram [3]

\subsection{Cold Quark Matter}

The Quark-Gluon Plasma is a state of QCD matter where quarks and gluons are deconfined. In figure 1.2 we can see a pictorial representation of the proton, which is constituted of three quarks, two up quarks and one down. Much like ice that melts when heated or pressurized, QCD has a similar melted state. If one heats or pressurizes enough a system of hadrons they will suffer a phase transition to a deconfined phase, that is what we call a Quark-Gluon Plasma (QGP). If you heat a nuclear system up to a critical temperature of $T_{c} \approx 150 \mathrm{MeV}$ at very low chemical potential, you will start to see effects of a deconfined phase which interacts very strongly with itself.

Experimentally we believe that we can produce this state of matter by colliding two heavy nuclei. Heavy ions (of Lead for instance) colliding with one another with high enough energy will penetrate each other, their nucleons will penetrate each other, and in the process will create a super hot state involving the constituents of those hadrons, the quarks and gluons, and many more other particles will be created during the collision.

This state, pictured in figure 1.3, is the hot QGP. This would be the analogous of heating the matter to create an ionized plasma of electrons, protons and neutrons. We also believe that a QGP can be formed in other systems 


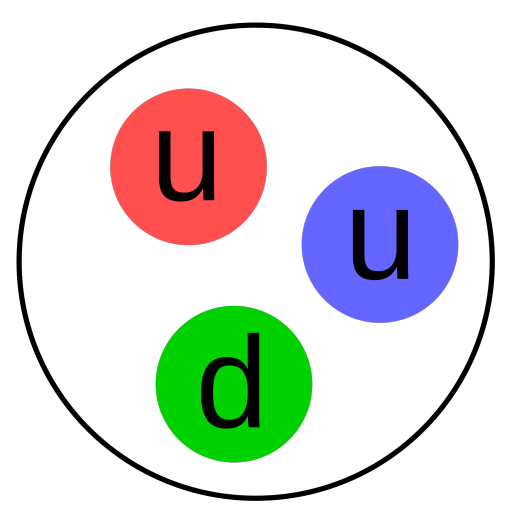

FIGURE 1.2: Proton structure of two quarks "up" and one "down"

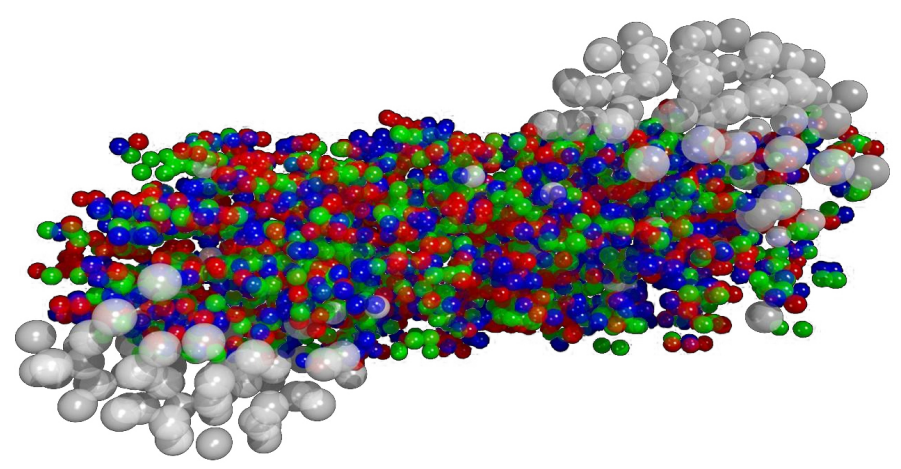

FIGURE 1.3: Illustration of Heavy Ion Collision. Image by Henning Weber / CERN

such as compact stars. However, inside a neutron star or a quark star the determinant factor creating the QGP is the pressure, not the temperature. That is why the state of matter inside the neutron star is commonly referred to as cold QGP (or cold quark matter). Figure 1.4 attempts to show the different ways of transitioning to a deconfined state. Heating the system will "melt the boundaries" of the hadrons, making the quarks and gluons deconfined, and pressurizing the system will make the boundaries overlap with each other and therefore the quarks will be able to move freely inside the plasma.

A very simple calculation, based on geometrical arguments, can be made to estimate at what density will the hadrons touch each other and therefore 
what is the critical density for this phase transition. Assuming that a nucleon is a sphere of $r_{N} \approx 1 \mathrm{fm}$, its volume is

$$
\frac{4 \pi r_{N}^{3}}{3}
$$

If the whole system has $N$ nucleons and its total volume is approximately just the sum of the nucleon volumes, i.e.

$$
N \times \frac{4 \pi r_{N}^{3}}{3}
$$

the number density of the system is

$$
N \times \frac{3}{4 \pi r_{N}^{3} \times N}
$$

which simplifies to

$$
\frac{3}{4 \pi r_{N}^{3}} \approx 0.24 \mathrm{fm}^{-3}=1.5 \rho_{0}
$$

where $\rho_{0}$ is the density of standard nuclear matter. Therefore, when the density reaches a value of around $\rho_{c}=0.24 \mathrm{fm}^{-3}$ we may expect that a QGP phase is about to be formed.
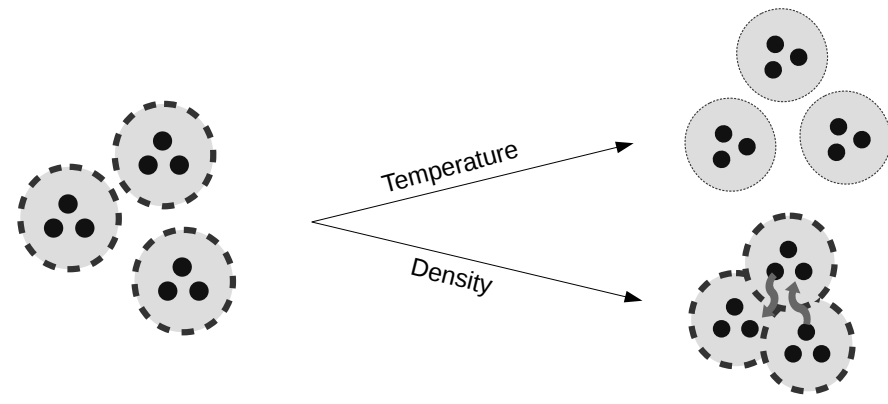

FIGURE 1.4: Deconfining quarks by increasing temperature or pressure

Until recently we believed that the QGP would be like a gas of partons (the particles that constitute a Hadron, i.e. quarks and gluons) in which the constituents barely interact with each other. However after many experiments colliding heavy ions we found out not only that the plasma has a surprisingly small viscosity but also that the plasma constituents interact 
very strongly with each other. Therefore this state known as the sQGP or strongly interacting QGP.

That is what we hope to find inside a neutron star core, or a quark star, a strongly interacting cold system made of gluons and quarks. The folowing dissertation exposes one particular model for the cold sQGP and its aplications to the astrophysics of compact objects.

\subsection{Neutron Stars}

As is shown in figure 1.1 there is a region of low temperature and high density (which is particularly higher than the expected critical density of phase transition) labeled neutron stars. According to our theoretical expectations the temperatures and densities of neutron stars are in that region.

\section{Stelar Evolution}

The formation of a star begins with a large gas dusty cloud in outer space, the nebulae, filled mostly with hydrogen. As the gravitational force pulls the cloud together and the density increases a stellar nursery filled with proto-stars is formed. Eventually the collapsing cloud will become denser and hotter and will start to fuse its hydrogen into helium. A full star is now combusting its hydrogen fuel into helium and emitting EM radiation. If the star is massive enough, other elements will be formed in a process called stellar nucleosynthesis. See ref. [4].

A dynamical equilibrium between the gravitational force, trying to pull the star together, and the thermal forces, trying to expand it, will take place. After a long enough time one of those forces dominates the other.

In the case of an average star the thermal force expands the star, cooling it and transforming it into a Red Giant. As the star cools the thermal forces weaken and the gravitational force once again pulls the star together. A long process will shed most of the star's mass to the surrounding space, leaving in the core a white dwarf (for more details see [4]).

In the case of a super massive star the cooling and expanding process will transform it into a Super Red Giant. However, when the gravitational 
forces crushes it back together, it will be a much more energetic event, in a much shorter time. This event is what is called a Supernova.

In a Supernova, the outer layer of the star is spilled over to outer space and its core becomes a neutron star, an extremely dense star, with masses of up to 2 solar masses but with the diameter of only a few kilometres.

If the initial star was massive enough the result of the supernova might not be a neutron star but rather a black hole.

If a neutron star is too dense, the neutrons will get squeezed against each other so strongly that they could break down into their constituents, the quarks and gluons predicted by QCD. Some theorists believe that a third type of compact stellar object (remainders of a stars collapse) might exist, the so called exotic stars or quark stars which are constituted of nothing but deconfined quarks.

Either in a quark star or a hybrid star core (a star with a deconfined quark phase in its core and a hadronic outer layer) we believe that a state of free quarks and gluons, the Quark Gluon Plasma, will exist, making the compact stars another laboratory for understanding QCD.

\subsection{Properties of Neutron Stars}

Neutron stars are part of the group of compact astrophysical objects. Those include white dwarfs, black holes, and of course neutron stars. Some objects that have not yet been proven to exist would also fit into this group such as quark stars, hybrid stars and strange stars (stable quark stars that contain the flavour $s$ ).

With radius of just tens of kilometers and masses of up to two solar masses, these objects can reach very high densities.

One very important characteristic of neutron stars is the magnetic field which in the center of the star is believed to reach values of around $10^{18} \mathrm{G}$ and maybe even higher. Amongst neutron stars with high magnetic fields many behave as pulsars. They usually have very high spins, sometimes reaching periods of rotation of the order of milliseconds. That spin combined with the unaligned magnetic field and the cloud of charged particles existing around the neutron star is what makes the star "pulsate", as an observer on Earth would see it. When the axes of the magnetic field points 
towards the observer a large amount of radiation is propagated and the star "blinks".

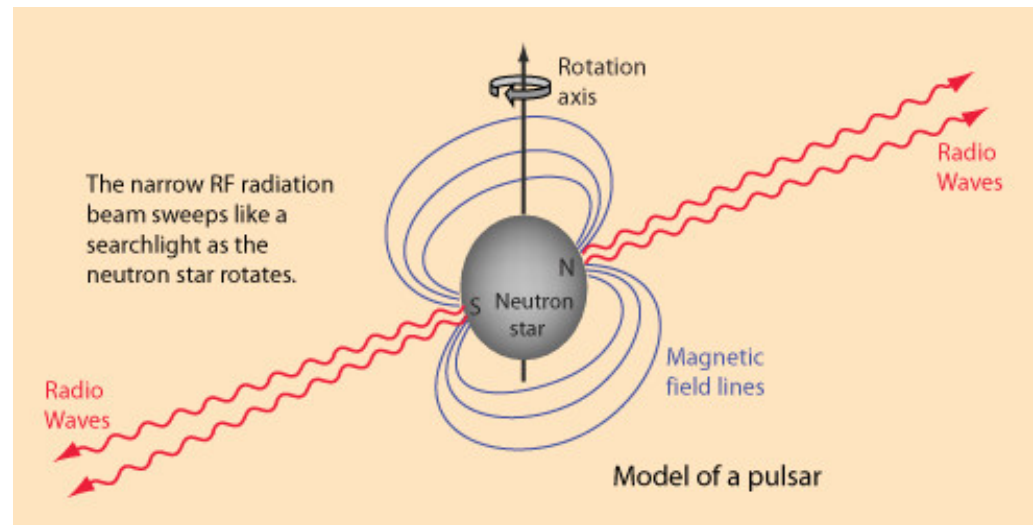

FIGURE 1.5: Pulsar illustration [5]

\subsubsection{Equilibrium \& Stability}

There are also some special conditions to the neutron star physical parameters. For instance, they are believed to be charge neutral. Since quarks are electrically charged and a small number of electrons is also present in the star, the abundance of these particles must be in charge equilibrium. I.e.

$$
\frac{2}{3} \rho_{u}=\frac{1}{3} \rho_{d}+\frac{1}{3} \rho_{s}+\rho_{e}
$$

Where $\rho$ represents the number densities of quarks $u, d$ and $s$ and electrons.

It is also believed to be in chemical equilibrium regarding all the possible decays and reactions of the quarks $[6,7]$ :

$$
\begin{gathered}
u+e^{-} \rightarrow d+\nu_{e}, \quad u+e^{-} \rightarrow s+\nu_{e}, \\
d \rightarrow u+e^{-}+\bar{\nu}_{e}, \quad s \rightarrow u+e^{-}+\bar{\nu}_{e}, \quad \text { and } \quad s+u \rightarrow d+u .
\end{gathered}
$$

The equilibrium provides the relations between the chemical potentials of the quarks up $\nu_{u}$, down $\nu_{d}$, strange $\nu_{u}$ and electrons $\nu_{e}$ :

$$
\nu_{d}=\nu_{s} \quad \text { and } \quad \nu_{u}+\mu_{e}=\nu_{s}
$$


Finally we must be sure that the physical parameters of the star are such that they properly describe a deconfined system. The energy per barionic number must not be too low so that the quarks would confine back into hadrons and it must not be high enough so that the system could produce new particles. The energy per baryonic number of the system with only quarks $u$ and $d$ must not be lower than the lowest energy per barionic number found in nuclei, which is $930 \mathrm{MeV}$ for iron. However, the presence of strange quarks corrects this value to $934 \mathrm{MeV}$ [6].

For the system with all three quark flavors, the energy must be lower than the nucleon mass as shown in [6] or else, the system could hadronize. This value is also corrected to $934 \mathrm{MeV}$ [6].

This leads to the condition

$$
\left.\frac{\varepsilon}{\rho_{B}}\right|_{(3 \text {-flavor })} \leq 934 \mathrm{MeV} \leq\left.\frac{\varepsilon}{\rho_{B}}\right|_{(2 \text {-flavor })}
$$

\subsubsection{Mass, Radius}

The most important parameters regarding neutron stars are the mass and the radius. Both are challengingly difficult to measure experimentally, but they provide very significant information regarding the interior of the star.

The first attempt to estimate the mass of such stars was made by Oppenheimer and Volkof [8]. They used an equation of state of a neutron gas and predicted a maximum mass of $0.7 M_{\odot}$. That calculation later became known as the Oppenheimer-Volkof limit. However, several neutron stars were determined experimentally to have masses much larger than that. Recent examples are the pulsars PSR J1614-2230, with $(1.97 \pm 0.04) M_{\odot}$ [9] or the pulsar PSR J0348+0432, with $(2.01 \pm 0.04) M_{\odot}[10]$ and perhaps the black widow pulsar PSR B1957+20, with a possible mass around $(2.4 \pm 0.12) M_{\odot}$ [11].

The larger the masses and densities we measure the more reasons we have to believe that a QGP phase must be present in the core of the star. 
TABLE 1.1: Different EOSs proposed. [12]

$\begin{array}{cc}\text { Authors } & \text { Composition } \\ \text { Pand e Smith (1976) } & n, \pi^{0} \\ \text { Friedman e Pandharipande(1981) } & n, p \\ \text { Muller, Prakash e Ainsworth (1987) } & n, p \\ \text { Wiringa, Fiks e Fabrocine (1988) } & n, p \\ \text { Prakash et al. (1988) } & n, p \\ \text { Glendenning e Moszkowski (1991) } & n, p, H \\ \text { Prakash, Cooke e Lattimer(1995) } & n, p, H, Q \\ \text { Prakash et al.(1995) } & Q \\ \text { Engvik et al.(1996) } & n, p \\ \text { Muller e Serot(1996) } & n, p \\ \text { Akmal e Pandharipande(1997) } & n, p \\ \text { Glendenning e Schaffner-Bielich(1999) } & n, p, K\end{array}$

The figure 1.6 shows a number of measured masses and radii of neutron stars. It is not simple to make a theoretical model to explain these measurements. In order to predict the mass and radius one has to know the equation of state (EOS) of the internal constituents of the star. The theoretical community has provided several different possible equations of state to describe the system, some of them include only quarks, some include also neutrons, hyperons, kaons, pions etc. A very illustrative table was depicted in [12] showing different equations of state. One reproduction of the same table is shown in Table 1.1.

Nowadays, after the measurement of the pulsars PSR J1614-2230 and PSR J0348+0432, every EOS has to predict stars with masses of at least two solar masses. In figure 1.7 we can see that several EOSs do cross the lines of those measurements. However, models with strange quark mater (SQM) yield EOSs that are usually too "soft" to support such high masses (this is not the case for every model, the color flavor locked model is one exception). 


\subsubsection{Magnetic Field \& Rotation}

Both magnetic field and rotation introduce an explicit symmetry breaking in an otherwise perfectly spherically symmetric and isotropic system. These aspects are, however, of huge importance to the system. First of all, there is a reasonable difference on the mass prediction of a theoretical model that includes the magnetic field and one that does not. The magnetic field seems to help the system to support significantly higher masses. This is a welcome feature as we can deduce from the discussions in the preceding section. Figure 1.8 shows the different types of neutron stars in a surface magnetic field vs period diagram. Although the magnetic field in the core of the star is much higher than the surface field, the figure illustrates well the big picture. Magnetars are of particular interest as they have very high magnetic fields. Figure 1.9 shows the stars in a $P \times \dot{P}$ diagram ( $P$ is the period and $\dot{P}$ is the time derivative of the period).

The magnetic field inside the core of a Magnetar is expected to be much higher than that of the average neutron star, reaching values of up to $10^{18} G$. Many studies were already made discussing the magnetic field and rotation effects on the stars $[15,16,17,18,19,20,21]$.

\subsubsection{Stellar Structure}

For non-compact stars the effects of general relativity on their internal structure are usually small. However, the study of the stellar structure of compact astrophysical objects has to be done in the relativistic framework. The equation governing the structure of a relativistic star are known as the TolmanOppenheimer-Volkof equations. They are one dimensional equations that describe the mass and pressure of the star as functions of only the radial variable (see [22] for equations involving angular variables as well):

$$
\begin{gathered}
\frac{d P(r)}{d r}=-\frac{1}{r^{2}}(\epsilon(r)+P(r))\left(M(r)+4 \pi r^{3} P(r)\right)\left(1-\frac{2 M(r)}{r}\right)^{-1} \\
\frac{d M(r)}{d r}=4 \pi r^{2} \epsilon(r) .
\end{gathered}
$$


where $M(r), P(r)$ and $\epsilon(r)$ are respectively the mass, pressure and energy density as functions of $r$.

These are the most simple equations to model the system. They are spherical, statical and isotropic. However, there are ways of studying more complex systems using these equations. One of this ways will be presented in Chapter 3. A full derivation of this equation is presented in Appendix A.

\subsection{Mean Field EOS with Magnetic Field}

As mentioned in section 1.3.2 several different equations of state have been proposed to explain the behavior of neutron stars and quark stars. One of these equatios was derived in [23] and is based on a technique called mean field approximation. We will refer to this equation of state as mean field QCD (mQCD).

The main purpose of this work is to include the magnetic field and rederive a similar EOS. The results presented in this dissertations were published in Ref. [1]. In this text we will discuss in detail the results of Ref. [1]. 


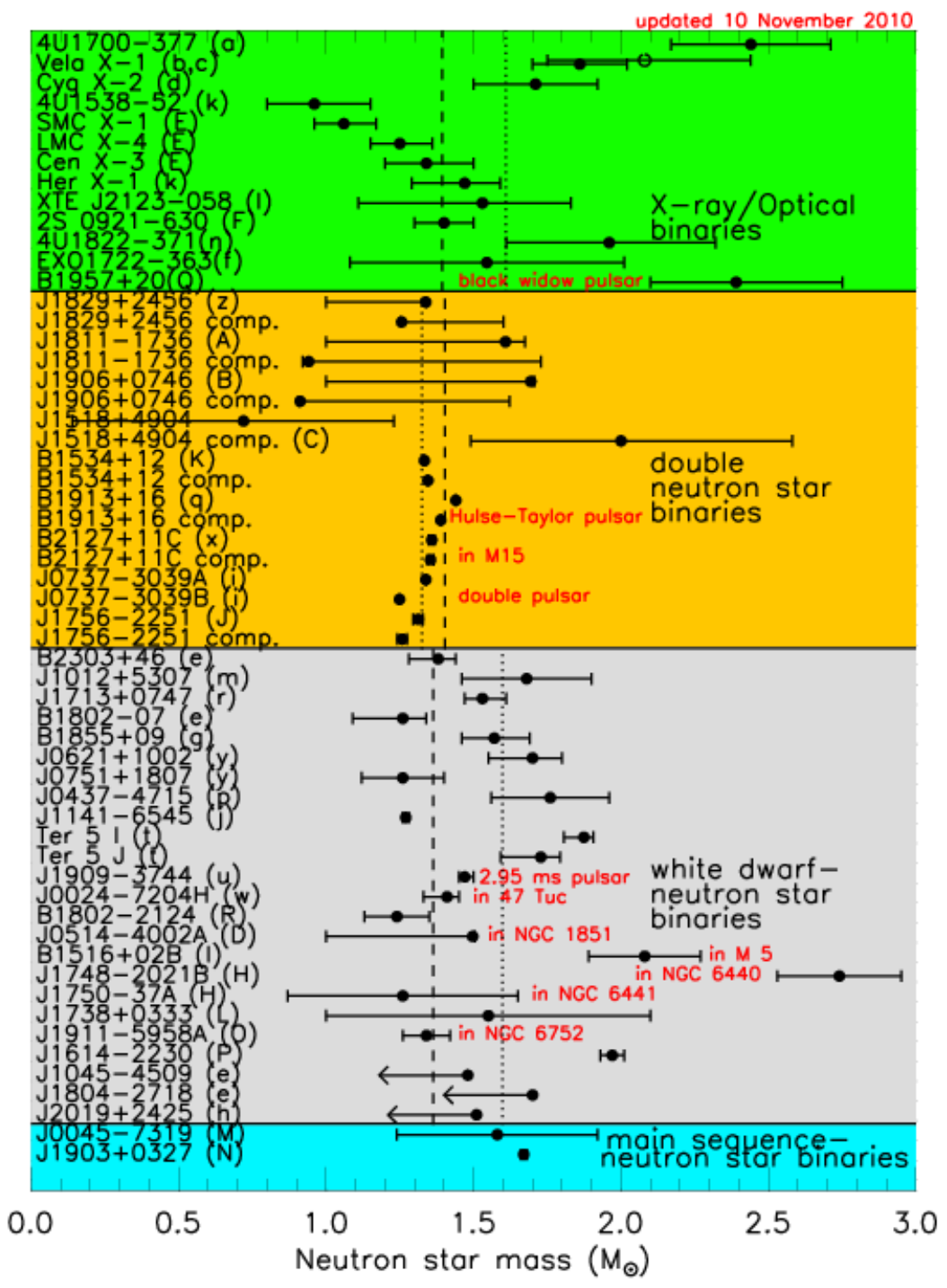

FIGURE 1.6: Measured NS masses [13] 


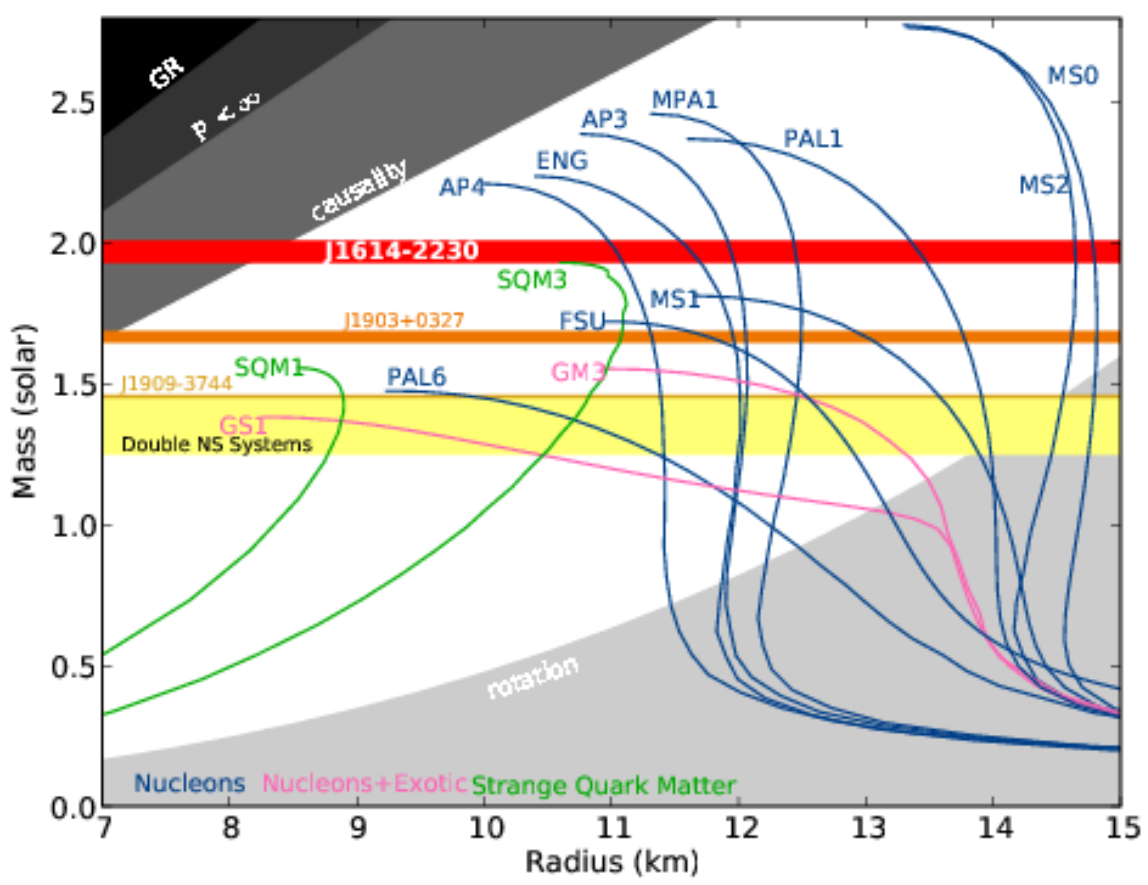

FIGURE 1.7: Different EOSs predictions for the Mass $\times$ Radius diagram of neutron stars [9] 


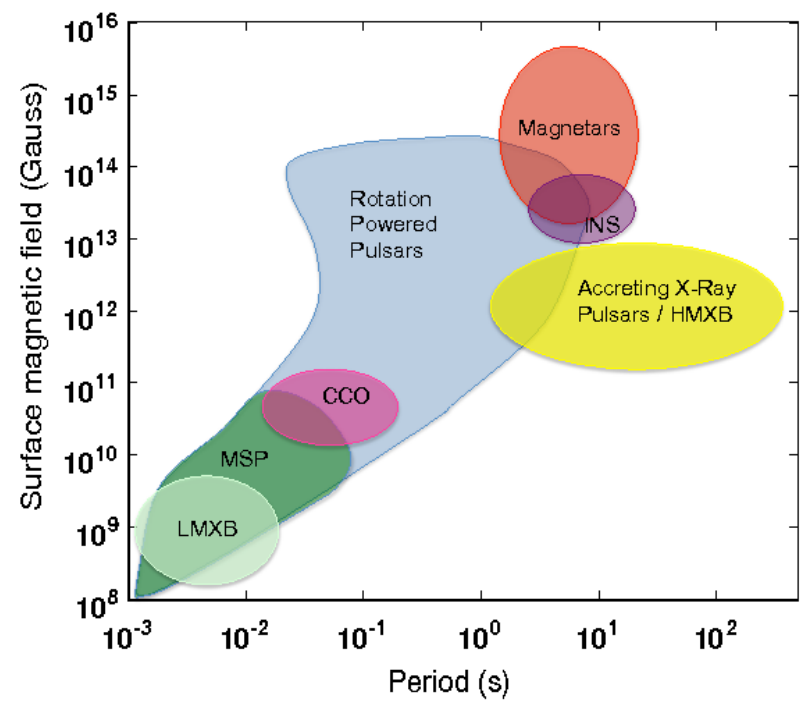

FIGURE 1.8: Different types of neutron star [14]. INS: Isolated Neutron Star. MSP: Millisecond Pulsars. L(H)MXB: Low (High) mass x-ray binaries. CCO: Compact central objects.

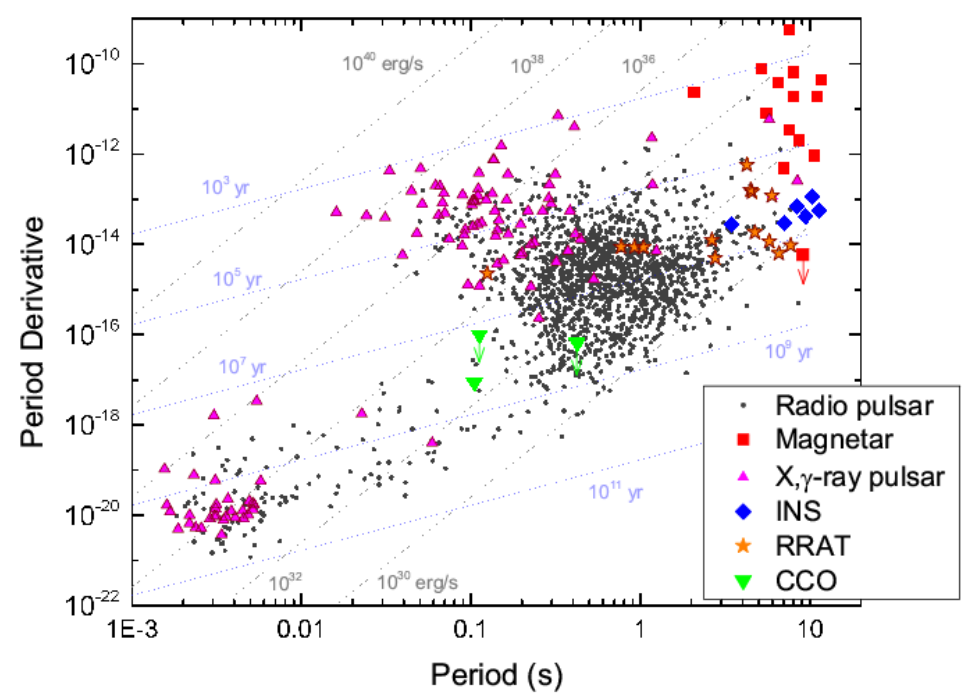

FiguRe 1.9: $P \times \dot{P}$ diagram [14] 


\section{Chapter 2}

\section{Effective Lagrangian and Equation of State}

In this chapter we derive the equation of state (EOS) of the QCD mean-fieldtheory model (mQCD). First, we perform the derivation of the EOS without the presence of the magnetic field and later we include it.

\subsection{QCD}

All the basics of QCD can be found in Ref.[2]. The QCD Lagrangian density is given by:

$$
\mathcal{L}_{Q C D}=-\frac{1}{4} F_{\mu \nu}^{a} F^{a \mu \nu}+\sum_{q=1}^{N_{f}} \bar{\psi}_{i}^{q}\left[i \gamma^{\mu}\left(\delta_{i j} \partial_{\mu}-i g T_{i j}^{a} G_{\mu}^{a}\right)-\delta_{i j} m\right] \psi_{j}^{q}
$$

where the sum in $q$ is performed over the quark flavors $N_{f}$. The Yang-Mills field tensor is given by:

$$
F^{a \mu \nu}=\partial^{\mu} G^{a \nu}-\partial^{\nu} G^{a \mu}+g f^{a b c} G^{b \mu} G^{c \nu}=\partial^{\mu} G^{a \nu}-\partial^{\nu} G^{a \mu}+g\left[G^{b \mu}, G^{c \nu}\right]
$$

and the generators of $\mathrm{SU}(3) T^{a}$ are given by:

$$
T^{a}=\frac{\lambda^{a}}{2} \quad a=1,2, \ldots, 8
$$


where the matrices " $\lambda$ " are the Gell-Mann matrices:

$$
\begin{gathered}
\lambda^{1}=\left(\begin{array}{lll}
0 & 1 & 0 \\
1 & 0 & 0 \\
0 & 0 & 0
\end{array}\right) \quad \lambda^{2}=\left(\begin{array}{ccc}
0 & -i & 0 \\
i & 0 & 0 \\
0 & 0 & 0
\end{array}\right) \quad \lambda^{3}=\left(\begin{array}{ccc}
1 & 0 & 0 \\
0 & -1 & 0 \\
0 & 0 & 0
\end{array}\right) \\
\lambda^{4}=\left(\begin{array}{lll}
0 & 0 & 1 \\
0 & 0 & 0 \\
1 & 0 & 0
\end{array}\right) \quad \lambda^{5}=\left(\begin{array}{ccc}
0 & 0 & -i \\
0 & 0 & 0 \\
i & 0 & 0
\end{array}\right) \quad \lambda^{6}=\left(\begin{array}{lll}
0 & 0 & 0 \\
0 & 0 & 1 \\
0 & 1 & 0
\end{array}\right) \\
\lambda^{7}=\left(\begin{array}{ccc}
0 & 0 & 0 \\
0 & 0 & -i \\
0 & i & 0
\end{array}\right) \quad \lambda^{8}=\frac{1}{\sqrt{3}}\left(\begin{array}{ccc}
1 & 0 & 0 \\
0 & 1 & 0 \\
0 & 0 & -2
\end{array}\right)
\end{gathered}
$$

and $f^{a b c}$ the anti-symmetrical structure constants of the $\mathrm{SU}(3)$ group.

$$
f^{b a c}=f^{a c b}=-f^{a b c}
$$

defined by the commutators:

$$
\left[\lambda^{a}, \lambda^{b}\right]=2 i f^{a b c} \lambda^{c}
$$

As each color index goes from 1 to 8 , the only non zero structure constants are the following:

$$
\begin{gathered}
f^{123}=1 \quad f^{147}=f^{246}=f^{257}=f^{345}=f^{516}=f^{637}=1 / 2 \\
f^{458}=f^{678}=\sqrt{3} / 2
\end{gathered}
$$

and consequently we get the following relations for $\mathrm{SU}(\mathrm{N})$ which will be useful:

$$
\begin{gathered}
f^{a b c} f^{a b c}=N\left(N^{2}-1\right) \stackrel{(\mathrm{N}=3)}{\longrightarrow} f^{a b c} f^{a b c}=24 \quad \text { and } \\
f^{a c d} f^{b c d}=N \delta^{a b} \stackrel{(\mathrm{N=3})}{\longrightarrow} f^{a c d} f^{b c d}=3 \delta^{a b}
\end{gathered}
$$




\subsection{Gluon Field Decomposition}

According to $[24,25]$ the decomposition of the gluon fields is given by:

$$
G^{a \mu}(k)=A^{a \mu}(k)+\alpha^{a \mu}(k)
$$

where $A^{a \mu}$ is the component of the field with only low momentum gluons (soft gluons) and $\alpha^{a \mu}$ is the component of high momentum gluons (hard gluons).

Considering (2.8) in the configuration space we have:

$$
G^{a \mu}(x)=A^{a \mu}(x)+\alpha^{a \mu}(x)
$$

where we note that $A^{a \mu}(x)$, which is the low momenta component, has very large wavelengths, therefore $\partial^{\nu} A^{a \mu}(x) \cong 0$. This is better understood from the Fourier Transform of $A^{a \mu}(k)$ :

$$
A^{a \mu}(x)=\int d^{4} k A^{a \mu}(k) e^{i k x}
$$

which makes its derivative:

$$
\partial^{\nu} A^{a \mu}(x)=\int d^{4} k i k^{\nu} A^{a \mu}(k) e^{i k x}
$$

Since $A^{a \mu}(k)$ is the low momenta component, we may assume that $k^{\nu} \cong 0$, and then the above expression is:

$$
\partial^{\nu} A^{a \mu}(x) \cong 0
$$

\subsection{Gluon Condensates}

We assume that, as in the case of the hot QGP produced in heavy ion collisions, the cold and deconfined quark matter is still in a strong coupling regime and therefore we can expect that gluon condensates will survive, even if with a reduced strength.

The contribution of the gluon condensates which is included in the model is one of the aspects that makes it different from the MIT Bag model. Accounting for the colourlessness of the vacuum we only consider condensates 
of dimension 2 and 4 .

Here we adopt the notation for expected values over the QGP simply as

$$
\left\langle Q G P_{T=0}|Y| Q G P_{T=0}\right\rangle \equiv\langle Y\rangle
$$

\subsubsection{Properties of the Condensates}

The important properties of this expectation values are:

- The colourlessness of the vacuum, which imposes:

$$
\begin{gathered}
\left\langle A^{a \mu}\right\rangle=0 \\
\left\langle A^{a \mu} A^{b \nu} A^{c \rho}\right\rangle=0
\end{gathered}
$$

- As calculated in $[24,25]$ we have:

$$
\left\langle A^{a \mu} A^{b \nu}\right\rangle=-\frac{\delta^{a b}}{8} \frac{g^{\mu \nu}}{4} \mu_{0}^{2}=-\frac{\delta^{a b} g^{\mu \nu}}{32} \mu_{0}^{2}
$$

where the factor of " 8 " is caused by the colour indices as they go from 1 to 8 and the factor of " 4 " is due to the 4-dimensions of Minkowsky space. As a consequence:

$$
\begin{gathered}
\left\langle A^{a \mu} A_{\mu}^{b}\right\rangle=\left\langle A^{a \mu} A_{\nu}^{b} g^{\nu}{ }_{\mu}\right\rangle=\left\langle A^{a \mu} A_{\nu}^{b}\right\rangle g^{\nu}{ }_{\mu}= \\
-\frac{\delta^{a b}}{32} g^{\mu}{ }_{\nu} g^{\nu}{ }_{\mu} \mu_{0}{ }^{2}=-\frac{\delta^{a b}}{32} 4 \times \mu_{0}{ }^{2}=-\frac{\delta^{a b}}{8} \mu_{0}{ }^{2}
\end{gathered}
$$

- From [25]:

$$
\left\langle A_{\mu}^{a} A_{\nu}^{b} A^{c \rho} A^{d \eta}\right\rangle=\frac{\phi_{0}{ }^{4}}{(32)(34)}\left[g_{\mu \nu} g^{\rho \eta} \delta^{a b} \delta^{c d}+g_{\mu}{ }^{\rho} g_{\nu}{ }^{\eta} \delta^{a c} \delta^{b d}+g_{\mu}{ }^{\eta} g_{\nu}{ }^{\rho} \delta^{a d} \delta^{b c}\right]
$$

and the factor "(32)(34)" in the denominator appears when we take $\mu=\nu, \rho=\eta, a=b$ e $c=d$ :

$$
\begin{gathered}
\left\langle A_{\mu}^{a} A_{\mu}^{a} A^{c \rho} A^{c \rho}\right\rangle=\phi_{0}{ }^{4}\left[g_{\mu \mu} g^{\rho \rho} \delta^{a a} \delta^{c c}+g_{\mu}{ }^{\rho} g_{\mu}{ }^{\rho} \delta^{a c} \delta^{a c}+g_{\mu}{ }^{\rho} g_{\mu}{ }^{\rho} \delta^{a c} \delta^{a c}\right] \\
=\phi_{0}{ }^{4}\left[16 \delta^{a a} \delta^{c c}+4 \delta^{a c} \delta^{a c}+4 \delta^{a c} \delta^{a c}\right]=\phi_{0}{ }^{4}[16 \times 8 \times 8+4 \times 8+4 \times 8]
\end{gathered}
$$




$$
=\phi_{0}{ }^{4}[4 \times 4 \times 8 \times 8+2 \times 4 \times 8]=\phi_{0}{ }^{4}(4 \times 8)(4 \times 8+2)=\phi_{0}{ }^{4}(32)(34)
$$

Also from [24, 25] we can define a dynamically generated gluon mass $m_{G}$ and the constant $b$ as:

$$
m_{G}^{2} \equiv\left(\frac{9}{2}\right)\left(\frac{1}{16}\right) g^{2} \mu_{0}^{2}
$$

and

$$
b \equiv \frac{9}{(4)(34)} g^{2}
$$

Those proprieties will be used in the derivations.

\subsection{Effective Lagrangian}

It is instructive to first derive a simplified version of the theory to get the basic results. Here we consider a non-magnetic plasma with two other simplifications: (i) the mass of the quarks $u, d, s$ will be the same $m$ for now. Later the sum over different quark masses will be considered, and (ii) the sum over the flavor indices will be ignored and later added again inside the degeneracy factor $\gamma_{Q}$ as a multiplicative factor 3. This makes our initial Lagrangian the following:

$$
\mathcal{L}_{Q C D}=-\frac{1}{4} F_{\mu \nu}^{a} F^{a \mu \nu}+\bar{\psi}_{i}\left[i \gamma^{\mu}\left(\delta_{i j} \partial_{\mu}-i g T_{i j}^{a} G_{\mu}^{a}\right)-\delta_{i j} m\right] \psi_{j}
$$

First we apply the gluon decomposition using (2.9) in (2.29) and in (2.2), and $\partial^{\nu} A^{a \mu}(x)=0$ :

$$
\begin{gathered}
\mathcal{L}_{Q C D}\left(G^{a \mu}=A^{a \mu}+\alpha^{a \mu}, \psi\right)=\mathcal{L}^{\prime}{ }_{Q C D} \\
\mathcal{L}^{\prime}{ }_{Q C D}=-\frac{1}{4} F_{\mu \nu}^{\prime a} F^{\prime a \mu \nu}+\bar{\psi}_{i}\left\{i \gamma^{\mu}\left[\delta_{i j} \partial_{\mu}-i g T_{i j}^{a}\left(A^{a \mu}+\alpha^{a \mu}\right)\right]-\delta_{i j} m\right\} \psi_{j}
\end{gathered}
$$

where $F^{a \mu \nu}=F^{a \mu \nu}\left(G^{a \mu}=A^{a \mu}+\alpha^{a \mu}\right)$ :

$$
\begin{gathered}
F^{\prime a \mu \nu}=g f^{a b c} A^{b \mu} A^{c \nu}+\left(\partial^{\mu} \alpha^{a \nu}-\partial^{\nu} \alpha^{a \mu}+g f^{a b c} \alpha^{b \mu} \alpha^{c \nu}\right) \\
+g f^{a b c} A^{b \mu} \alpha^{c \nu}+g f^{a b c} \alpha^{b \mu} A^{c \nu}
\end{gathered}
$$


next we carry out the distributive multiplications that turn the lagrangian into:

$$
\begin{gathered}
\mathcal{L}^{\prime}{ }_{Q C D}=-\frac{1}{4} \Gamma_{\mu \nu}^{a} \Gamma^{a \mu \nu}-\frac{g f^{a b c}}{2} \Gamma_{\mu \nu}^{a}\left(A^{b \mu} \alpha^{c \nu}+\alpha^{b \mu} A^{c \nu}+A^{b \mu} A^{c \nu}\right) \\
-\frac{g^{2} f^{a b c} f^{a d e}}{4}\left[A_{\mu}^{b} A_{\nu}^{c} A^{d \mu} A^{e \nu}\right. \\
+A_{\mu}^{b} A_{\nu}^{c} A^{d \mu} \alpha^{e \nu}+A_{\mu}^{b} A_{\nu}^{c} \alpha^{d \mu} A^{e \nu}+A_{\mu}^{b} \alpha_{\nu}^{c} A^{d \mu} A^{e \nu}+\alpha_{\mu}^{b} A_{\nu}^{c} A^{d \mu} A^{e \nu} \\
\left.+A_{\mu}^{b} \alpha_{\nu}^{c} \alpha^{d \mu} A^{e \nu}+A_{\mu}^{b} \alpha_{\nu}^{c} A^{d \mu} \alpha^{e \nu}+\alpha_{\mu}^{b} A_{\nu}^{c} A^{d \mu} \alpha^{e \nu}+\alpha_{\mu}^{b} A_{\nu}^{c} \alpha^{d \mu} A^{e \nu}\right] \\
+\bar{\psi}_{i}\left\{i \gamma^{\mu}\left[\delta_{i j} \partial_{\mu}-i g T_{i j}^{a}\left(A_{\mu}^{a}+\alpha_{\mu}^{a}\right)\right]-\delta_{i j} m\right\} \psi_{j}
\end{gathered}
$$

where

$$
\Gamma^{a \mu \nu}=\partial^{\mu} \alpha^{a \nu}-\partial^{\nu} \alpha^{a \mu}+g f^{a b c} \alpha^{b \mu} \alpha^{c \nu}
$$

We now, substitute the terms involving soft gluons by their expectation values:

$$
\begin{gathered}
\mathcal{L}^{\prime}{ }_{Q C D}=-\frac{1}{4} \Gamma_{\mu \nu}^{a} \Gamma^{a \mu \nu}-\frac{g f^{a b c}}{2} \Gamma_{\mu \nu}^{a}\left(\left\langle A^{b \mu}\right\rangle \alpha^{c \nu}+\alpha^{b \mu}\left\langle A^{c \nu}\right\rangle+\left\langle A^{b \mu} A^{c \nu}\right\rangle\right) \\
-\frac{g^{2} f^{a b c} f^{a d e}}{4}\left[\left\langle A_{\mu}^{b} A_{\nu}^{c} A^{d \mu} A^{e \nu}\right\rangle\right. \\
+\left\langle A_{\mu}^{b} A_{\nu}^{c} A^{d \mu} \alpha^{e \nu}\right\rangle+\left\langle A_{\mu}^{b} A_{\nu}^{c} \alpha^{d \mu} A^{e \nu}\right\rangle+\left\langle A_{\mu}^{b} \alpha_{\nu}^{c} A^{d \mu} A^{e \nu}\right\rangle+\left\langle\alpha_{\mu}^{b} A_{\nu}^{c} A^{d \mu} A^{e \nu}\right\rangle \\
\left.+\left\langle A_{\mu}^{b} \alpha_{\nu}^{c} \alpha^{d \mu} A^{e \nu}\right\rangle+\left\langle A_{\mu}^{b} \alpha_{\nu}^{c} A^{d \mu} \alpha^{e \nu}\right\rangle+\left\langle\alpha_{\mu}^{b} A_{\nu}^{c} A^{d \mu} \alpha^{e \nu}\right\rangle+\left\langle\alpha_{\mu}^{b} A_{\nu}^{c} \alpha^{d \mu} A^{e \nu}\right\rangle\right] \\
+\bar{\psi}_{i}\left\{i \gamma^{\mu}\left[\delta_{i j} \partial_{\mu}-i g T_{i j}^{a}\left(\left\langle A_{\mu}^{a}\right\rangle+\alpha_{\mu}^{a}\right)\right]-\delta_{i j} m\right\} \psi_{j}
\end{gathered}
$$

And using the condensate (2.10 to 2.14) we get:

$$
\begin{gathered}
\mathcal{L}^{\prime}{ }_{Q C D}=-\frac{1}{4} \Gamma_{\mu \nu}^{a} \Gamma^{a \mu \nu}+\frac{g f^{a b b}}{2} \Gamma_{\mu \nu}^{a} \frac{g^{\mu \nu}}{32} \mu_{0}{ }^{2} \\
-\frac{g^{2}}{4} \frac{\phi_{0}{ }^{4}}{(32)(34)}\left[g_{\mu \nu} g^{\mu \nu} f^{a b b} f^{a d d}+g_{\mu}{ }^{\mu} g_{\nu}{ }^{\nu} f^{a b c} f^{a b c}+g_{\mu}{ }^{\nu} g_{\nu}{ }^{\mu} f^{a b c} f^{a c b}\right] \\
+\frac{g^{2} f^{a c b} f^{a e d}}{4} \alpha_{\nu}^{c}\left(\frac{\delta^{b e} g_{\mu}{ }^{\nu}}{32} \mu_{0}{ }^{2}\right) \alpha^{d \mu}-\frac{g^{2} f^{a c b} f^{a d e}}{4} \alpha_{\nu}^{c}\left(\frac{\delta^{b d}}{8} \mu_{0}{ }^{2}\right) \alpha^{e \nu}
\end{gathered}
$$




$$
\begin{gathered}
+\frac{g^{2} f^{a b c} f^{a d e}}{4} \alpha_{\mu}^{b}\left(\frac{\delta^{c d} g_{\nu}{ }^{\mu}}{32} \mu_{0}^{2}\right) \alpha^{e \nu}-\frac{g^{2} f^{a b c} f^{a e d}}{4} \alpha_{\mu}^{b}\left(\frac{\delta^{c e}}{8} \mu_{0}^{2}\right) \alpha^{d \mu} \\
+\bar{\psi}_{i}\left[i \gamma^{\mu}\left(\delta_{i j} \partial_{\mu}-i g T_{i j}^{a} \alpha_{\mu}^{a}\right)-\delta_{i j} m\right] \psi_{j}
\end{gathered}
$$

Contracting the metric tensors and using the anti-symmetry of the structure factors we get:

$$
\begin{aligned}
& \mathcal{L}^{\prime}{ }_{Q C D}=-\frac{1}{4} \Gamma_{\mu \nu}^{a} \Gamma^{a \mu \nu}-\frac{g^{2}}{4} \frac{\phi_{0}{ }^{4}}{(32)(34)}\left[16 f^{a b c} f^{a b c}+4 f^{a b c} f^{a c b}\right] \\
&+ \frac{g^{2} f^{a c b} f^{a b d}}{4} \alpha_{\nu}^{c} \alpha^{d \nu} \frac{\mu_{0}^{2}}{32}-\frac{g^{2} f^{a c b} f^{a b e}}{4} \alpha_{\nu}^{c} \alpha^{e \nu} \frac{\mu_{0}^{2}}{8} \\
&+ \frac{g^{2} f^{a b c} f^{a c e}}{4} \alpha_{\mu}^{b} \alpha^{e \mu} \frac{\mu_{0}^{2}}{32}-\frac{g^{2} f^{a b c} f^{a c d}}{4} \alpha_{\mu}^{b} \alpha^{d \mu} \frac{\mu_{0}^{2}}{8} \\
&+\bar{\psi}_{i}\left[i \gamma^{\mu}\left(\delta_{i j} \partial_{\mu}-i g T_{i j}^{a} \alpha_{\mu}^{a}\right)-\delta_{i j} m\right] \psi_{j}
\end{aligned}
$$

Using (2.7) and opening the term $-\frac{1}{4} \Gamma_{\mu \nu}^{a} \Gamma^{a \mu \nu}$ we end up with the following Lagrangian:

$$
\begin{gathered}
\mathcal{L}^{\prime}{ }_{Q C D}=-\frac{1}{2}\left[\left(\partial_{\mu} \alpha_{\nu}^{a}\right) \partial^{\mu} \alpha^{a \nu}-\left(\partial_{\nu} \alpha_{\mu}^{a}\right) \partial^{\mu} \alpha^{a \nu}\right]-\frac{1}{4}\left[\left(\partial_{\mu} \alpha_{\nu}^{a}-\partial_{\nu} \alpha_{\mu}^{a}\right) g f^{a b c} \alpha^{b \mu} \alpha^{c \nu}\right. \\
\left.+g f^{a b c} \alpha_{\mu}^{b} \alpha_{\nu}^{c}\left(\partial^{\mu} \alpha^{a \nu}-\partial^{\nu} \alpha^{a \mu}\right)\right]-\frac{g^{2} f^{a b c} f^{a d e}}{4} \alpha_{\mu}^{b} \alpha_{\nu}^{c} \alpha^{d \mu} \alpha^{e \nu} \\
-\frac{9}{(4)(34)} g^{2} \phi_{0}{ }^{4}+\left(\frac{9}{4}\right)\left(\frac{1}{16}\right) g^{2} \mu_{0}{ }^{2} \alpha_{\nu}^{a} \alpha^{a \nu} \\
+\bar{\psi}_{i}\left[i \gamma^{\mu}\left(\delta_{i j} \partial_{\mu}-i g T_{i j}^{a} \alpha_{\mu}^{a}\right)-\delta_{i j} m\right] \psi_{j}
\end{gathered}
$$

Looking closely to the action of this theory we see that one surface term is canceled.

The action is:

$$
S_{Q C D}^{\prime}=\int d^{4} x \mathcal{L}^{\prime}{ }_{Q C D}
$$

and the contribution of the first term in (2.23) when integrated is:

$$
\begin{gathered}
-\frac{1}{2} \int d^{4} x\left[\left(\partial_{\mu} \alpha_{\nu}^{a}\right) \partial^{\mu} \alpha^{a \nu}-\left(\partial_{\nu} \alpha_{\mu}^{a}\right) \partial^{\mu} \alpha^{a \nu}\right] \\
=-\left.\frac{1}{2}\left[\alpha_{\nu}^{a}\left(\partial^{\mu} \alpha^{a \nu}\right)-\alpha_{\mu}^{a}\left(\partial^{\mu} \alpha^{a \nu}\right)\right]\right|_{-\infty} ^{+\infty}+\frac{1}{2} \int d^{4} x\left[\alpha_{\nu}^{a}\left(\partial_{\mu} \partial^{\mu} \alpha^{a \nu}\right)-\alpha_{\mu}^{a}\left(\partial_{\nu} \partial^{\mu} \alpha^{a \nu}\right)\right]
\end{gathered}
$$




$$
=\frac{1}{2} \int d^{4} x\left[\alpha_{\nu}^{a}\left(\partial_{\mu} \partial^{\mu} \alpha^{a \nu}\right)-\alpha_{\mu}^{a}\left(\partial_{\nu} \partial^{\mu} \alpha^{a \nu}\right)\right]
$$

where the surface term is zero due to the fact that we will not consider extended field configurations. Then, rewriting (2.23) we get

$$
\begin{gathered}
\mathcal{L}_{Q C D}^{\prime}=\frac{1}{2}\left[\alpha_{\nu}^{a}\left(\partial_{\mu} \partial^{\mu} \alpha^{a \nu}\right)-\alpha_{\mu}^{a}\left(\partial_{\nu} \partial^{\mu} \alpha^{a \nu}\right)\right]-\frac{1}{4}\left[\left(\partial_{\mu} \alpha_{\nu}^{a}-\partial_{\nu} \alpha_{\mu}^{a}\right) g f^{a b c} \alpha^{b \mu} \alpha^{c \nu}\right. \\
\left.+g f^{a b c} \alpha_{\mu}^{b} \alpha_{\nu}^{c}\left(\partial^{\mu} \alpha^{a \nu}-\partial^{\nu} \alpha^{a \mu}\right)\right]-\frac{g^{2} f^{a b c} f^{a d e}}{4} \alpha_{\mu}^{b} \alpha_{\nu}^{c} \alpha^{d \mu} \alpha^{e \nu} \\
-\frac{9}{(4)(34)} g^{2} \phi_{0}{ }^{4}+\left(\frac{9}{4}\right)\left(\frac{1}{16}\right) g^{2} \mu_{0}{ }^{2} \alpha_{\nu}^{a} \alpha^{a \nu} \\
+\bar{\psi}_{i}\left[i \gamma^{\mu}\left(\delta_{i j} \partial_{\mu}-i g T_{i j}^{a} \alpha_{\mu}^{a}\right)-\delta_{i j} m\right] \psi_{j}
\end{gathered}
$$

Here we apply the mean field approximation to the hard gluon fields. Assuming the occupation number of the hard gluons is large (because the quark sources have high density and the coupling is not small) and the gluon field can be assumed to be a classical field, a Walecka-like approximation is done [26, 27] as already applied to QCD in [28, 29]:

$$
\alpha_{\mu}^{a}=\alpha_{0}^{a} \delta_{\mu 0}
$$

where $\alpha_{0}^{a}=\alpha_{0}^{a}(\vec{x}, t)$ is a classical field.

The Lagrangian (2.25) becomes then:

$$
\begin{gathered}
\mathcal{L}_{0}=\frac{1}{2}\left[\alpha_{0}^{a}\left(\partial_{\mu} \partial^{\mu} \alpha_{0}^{a}\right)-\alpha_{0}^{a}\left(\partial_{0} \partial^{0} \alpha_{0}^{a}\right)\right] \\
-\frac{1}{4}\left[\left(\partial_{0} \alpha_{0}^{a}-\partial_{0} \alpha_{0}^{a}\right) g f^{a b c} \alpha_{0}^{b} \alpha_{0}^{c}+g f^{a b c} \alpha_{0}^{b} \alpha_{0}^{c}\left(\partial^{0} \alpha_{0}^{a}-\partial^{0} \alpha_{0}^{a}\right)\right]-\frac{g^{2} f^{a b c} f^{a d e}}{4} \alpha_{0}^{b} \alpha_{0}^{c} \alpha_{0}^{d} \alpha_{0}^{e} \\
-\frac{9}{(4)(34)} g^{2} \phi_{0}{ }^{4}+\left(\frac{9}{4}\right)\left(\frac{1}{16}\right) g^{2} \mu_{0}{ }^{2} \alpha_{0}^{a} \alpha_{0}^{a} \\
+\bar{\psi}_{i}\left[i \gamma^{\mu}\left(\delta_{i j} \partial_{\mu}-i g T_{i j}^{a} \alpha_{0}^{a} \delta_{\mu 0}\right)-\delta_{i j} m\right] \psi_{j}
\end{gathered}
$$

Since $f^{a b c} f^{a d e}$ and $\alpha_{0}^{b} \alpha_{0}^{c} \alpha_{0}^{d} \alpha_{0}^{e}$ are anti-symmetric and symmetric tensors respectively, the multiplication results in zero. Also using the definitions (2.15) and (2.16) we are left with the final lagrangian: 


$$
\mathcal{L}_{0}=-\frac{1}{2} \alpha_{0}^{a}\left(\vec{\nabla}^{2} \alpha_{0}^{a}\right)+\frac{m_{G}{ }^{2}}{2} \alpha_{0}^{a} \alpha_{0}^{a}-b \phi_{0}{ }^{4}+\bar{\psi}_{i}\left(i \delta_{i j} \gamma^{\mu} \partial_{\mu}+g \gamma^{0} T_{i j}^{a} \alpha_{0}^{a}-\delta_{i j} m\right) \psi_{j}
$$

We also usually take, for simplicity, the hard gluon field as a constant field $\alpha_{0}^{a}=$ const. For our purposes the terms involving non-homogeneity aspects of the field are not relevant. This was relevant in the study of wave propagations through the plasma [30,31].

And as we can see not much was done with the quark term, so to return to the case of different quark masses we can just perform the sum over flavors and add an index in the mass term.

The final Lagrangian is:

$$
\mathcal{L}_{0}=\frac{m_{G}^{2}}{2} \alpha_{0}^{a} \alpha_{0}^{a}-b \phi_{0}^{4}+\sum_{f=u, d, s} \bar{\psi}_{i}^{f}\left(i \delta_{i j} \gamma^{\mu} \partial_{\mu}+g \gamma^{0} T_{i j}^{a} \alpha_{0}^{a}-\delta_{i j} m_{f}\right) \psi_{j}^{f}
$$

\subsection{Effective Lagrangian with Magnetic Field}

Now that we have derived the mQCD Lagrangian we can more easily derive the more complicated version of the model. This version consists on adding the following parameters to our system:

1. A classic magnetic field, constant in magnitude and direction. $B=B \hat{z}$, i.e. $A_{\mu}=(0, y B, 0,0)$

2. Another spinorial field representing the electrons whose eletrical charges will later be necessary to guarantee the neutrality of charge in our compact stars.

The new Lagrangian is:

$$
\begin{gathered}
\mathcal{L}=-\frac{1}{4} F_{\mu \nu}^{a} F^{a \mu \nu}+\sum_{f=u}^{d, s} \bar{\psi}_{i}^{f}\left[i \gamma^{\mu}\left(\delta_{i j} \partial_{\mu}+i \delta_{i j} Q_{f} A_{\mu}-i g T_{i j}^{a} G_{\mu}^{a}\right)-\delta_{i j} m_{f}\right] \psi_{j}^{f} \\
-\frac{1}{16 \pi} F_{\mu \nu} F^{\mu \nu}+\bar{\psi}_{i}^{e}\left[i \gamma^{\mu}\left(\delta_{i j} \partial_{\mu}+i \delta_{i j} Q_{e} A_{\mu}\right)-\delta_{i j} m_{e}\right] \psi_{j}^{e}
\end{gathered}
$$


where $Q_{f}, Q_{e}$, and $m_{e}$ are the $f$ flavored quark charge, the electron charge and the electron mass respectively.

We do not need to do the derivations again. Note that since the gluon field does not couple to the electromagnetic field, the same series of approximations on this field will eventually end up in the same contribution to the Lagrangian

$$
\frac{m_{G}{ }^{2}}{2} \alpha_{0}^{a} \alpha_{0}^{a}-b \phi_{0}{ }^{4}
$$

Moreover, the influence of the magnetic field on the quark term of the Lagrangian will be simply:

$$
\sum_{f=u, d, s} \bar{\psi}_{i}^{f}\left(i \gamma^{\mu}\left[\delta_{i j} \partial_{\mu}+i \delta_{i j} Q_{f} A_{\mu}\right]+g \gamma^{0} T_{i j}^{a} \alpha_{0}^{a}-\delta_{i j} m_{f}\right) \psi_{j}^{f}
$$

Also, since no approximations will be done in the electric term in the final approximate Lagrangian this term will have suffered no change from the initial Lagrangian. So all we have to calculate to find the approximate Lagrangian with the magnetic field is the magnetic term $-\frac{1}{16 \pi} F_{\mu \nu} F^{\mu \nu}$. This is just the total energy contribution which we get from the most basic classical electrodynamics:

$$
-\frac{1}{16 \pi} F_{\mu \nu} F^{\mu \nu}=-\frac{B^{2}}{8 \pi}
$$

The transition to the magnetic case is then just a matter of adding this term and the vector potential influence on the spinorial fields:

$$
\begin{aligned}
\mathcal{L}_{0} & =\frac{m_{G}{ }^{2}}{2} \alpha_{0}^{a} \alpha_{0}^{a}-b \phi_{0}{ }^{4}-\frac{B^{2}}{8 \pi}+\bar{\psi}_{i}^{e}\left[i \gamma^{\mu}\left(\delta_{i j} \partial_{\mu}+i \delta_{i j} Q_{e} A_{\mu}\right)-\delta_{i j} m_{e}\right] \psi_{j}^{e} \\
& +\sum_{f=u}^{d, s} \bar{\psi}_{i}^{f}\left\{i \gamma^{\mu}\left[\delta_{i j} \partial_{\mu}+i \delta_{i j} Q_{f} A_{\mu}\right]+g_{h} \gamma^{0} T_{i j}^{a} \alpha_{0}^{a}-\delta_{i j} m_{f}\right\} \psi_{j}^{f}
\end{aligned}
$$

We can recover the first, non-magnetic Lagrangian, just by taking the vector potential and the magnetic field to be zero. 


\subsubsection{Equations of Motion}

Although our main goal is to obtain the energy-momentum tensor and from that the EOS, the equations of motion have some interesting properties that we should look into before going further.

The Euler-Lagrange equations are

$$
\frac{\partial \mathcal{L}}{\partial \varphi_{i}}-\partial_{\mu} \frac{\partial \mathcal{L}}{\partial\left(\partial_{\mu} \varphi_{i}\right)}=0
$$

So for each respective field (electrons, quarks, gluons and EM) we have the following equations of motion:

$$
\begin{gathered}
{\left[i \gamma^{\mu}\left(\partial_{\mu}+i Q_{f} A_{\mu}\right)+g_{h} \gamma^{0} T^{a} \alpha_{0}^{a}-m_{f}\right] \psi^{f}=0} \\
{\left[i \gamma^{\mu}\left(\partial_{\mu}+i Q_{e} A_{\mu}\right)-m_{e}\right] \psi^{e}=0} \\
m_{G}^{2} \alpha_{0}^{a}=-g_{h} \sum_{f=u}^{d, s} \rho_{f}^{a}=-g_{h} \rho^{a} \\
\partial_{\mu} F^{\mu \nu}=\sum_{f=u}^{d, s} Q_{f}\left(\bar{\psi}^{f} \gamma^{\nu} \psi^{f}\right)+Q_{e}\left(\bar{\psi}^{e} \gamma^{\nu} \psi^{e}\right)
\end{gathered}
$$

where $\rho^{a}$ is the charge density of color $a$. It is given by the temporal component of the color density current:

$$
j^{a 0}=\rho^{a}=\sum_{f=u}^{d, s} \bar{\psi}_{i}^{f} \gamma^{0} T_{i j}^{a} \psi_{j}^{f}=\sum_{f=u}^{d, s} \psi_{i}^{\dagger} T_{i j}^{a} \psi_{j}^{f}
$$

\subsubsection{Landau Levels}

The most important feature of the equations of motion is the existence of Landau levels. There is now a quantum number associated to the magnetic field relation with the charged particle orbits.

\section{Exact Solution}

Let us consider the electronic equation (2.33) omitting the electronic indices for now. If we multiply it by $\gamma^{0}$ from the left we can use the relation $\gamma_{0} \gamma_{0}=1$ 
and write it in a usual eigenfunction form (see [32])

$$
i \partial_{t} \psi=H_{\text {Dirac }} \psi
$$

where $H_{\text {Dirac }}=\sigma^{i}\left(-i \partial_{i}-Q_{e} A_{i}\right)+\gamma^{0} m_{e}$, and $\sigma^{i}$ is just $\gamma^{0} \gamma^{i}$. From now on we will choose these $\sigma$ matrices as the Pauli matrices and $\gamma_{0}=\operatorname{diag}(1,1,-1,-1)$. So, its functional form is clearly

$$
\psi=e^{-i E t}\left(\begin{array}{c}
\phi \\
\xi
\end{array}\right)
$$

where the spinor is divided into two components of two components.

The equations for them are:

$$
\begin{aligned}
& (E-m) \phi=\sigma^{i}\left(-i \nabla_{i}-e Q A_{i}\right) \xi \\
& (E+m) \xi=\sigma^{i}\left(-i \nabla_{i}-e Q A_{i}\right) \phi
\end{aligned}
$$

and we can isolate one component, e.g.

$$
\left(E^{2}-m^{2}\right) \phi=\left(\sigma^{i}\left[-i \nabla_{i}-e Q A_{i}\right]\right)^{2} \phi
$$

Substituting the vector potential, we obtain:

$$
\left(E^{2}-m^{2}\right) \phi=\left(-\nabla^{2}+[e Q B]^{2} y^{2}-e Q B\left[2 i y \partial_{x}+\sigma_{3}\right]\right) \phi
$$

The absence of $x$ and $z$ components except through derivatives suggests a functional form for the result:

$$
\phi=e^{i \mathbf{k} \cdot \mathbf{x}} f(y)
$$

where $f(y)$ is a two component function and $X$ is the spatial vector. Two linear independent solutions appear

$$
f_{+}=\left(\begin{array}{c}
F_{+}(y) \\
0
\end{array}\right) \text { and } f_{-}=\left(\begin{array}{c}
0 \\
F_{-}(y)
\end{array}\right)
$$

We want these $f$ functions to be eigenfunctions of $\sigma_{3}$ with eigenvalues $s$ and hence: 


$$
\partial_{y}^{2} F_{s}=\left(q Q B y+k_{x}\right)^{2} F_{s}-\left(E^{2}-m^{2}-k_{z}^{2}+e Q B s\right) F_{s}
$$

which is in fact a Hermite equation. If we changes the variables as

$$
\chi=\sqrt{e|Q| B}\left(y+\frac{k_{x}}{e Q B}\right)
$$

we get the equation $\left(\partial_{\chi}^{2}-\chi^{2}+a_{s}\right) F_{s}=0$ with

$$
a_{s}=\frac{E^{2}-m^{2}-k_{z}^{2}+e Q B s}{e|Q| B}
$$

Finally we obtain the energy eigenvalues

$$
E_{\nu}^{2}=m^{2}+k_{z}^{2}+(2 \nu+1) e|Q| B-e Q B s
$$

\section{Quarks}

We could do everything again for the quarks but if we notice that the difference between the two equations of motion (2.33) and (2.32) is just a new constant term in the Hamiltonian $H \rightarrow H+H^{\prime}(C t e)$ we can just use the eigenvalue properly $E \rightarrow E+E^{\prime}$.

The only major diference between the two equations is that the quark spinor has a color index accounted by a $c_{j}$ matrix multiplying eq (2.37) where $c_{i} c_{j}=\delta_{i j}$ so all is resolved multiplying equations (2.38) by a $c_{j}$ matrix from the left. If we redefine the energy variable according to

$$
\tilde{E}_{\nu}^{f} \equiv E_{\nu}^{f}+g_{h} \mathcal{A}
$$

Where $\mathcal{A}=c_{i}^{\dagger} T_{i j}^{a} c_{j} \alpha_{0}^{a}$ we are left with the usual eigenvalues for the Dirac equation in the presence of a constant magnetic field. So we isolate the energy as usual and the solution of (2.32) gives us the energy eigenvalues:

$$
\left(E_{\nu}^{f}+g_{h} \mathcal{A}\right)^{2}=m_{f}^{2}+k_{z}^{2}+(2 \nu+1)\left|Q_{f}\right| B-Q_{f} B s
$$

where the Hermite number is $\nu=0,1,2,3, \ldots$ and the spin is $s= \pm 1$. Isolating the energy, the above equation becomes 


$$
\left(\tilde{E}_{\nu}^{f}\right)^{2}=m_{f}^{2}+k_{z}^{2}+\left[2 \nu+1-s \times \operatorname{sign}\left(Q_{f}\right)\right]\left|Q_{f}\right| B
$$

and redefining the quantum number we have

$$
\tilde{E}_{n}^{f( \pm)}= \pm \sqrt{m_{f}^{2}+k_{z}^{2}+2 n\left|Q_{f}\right| B}
$$

where the Landau quantum number is $2 n=2 \nu+1-s \times \operatorname{sign}\left(Q_{f}\right)$.

We find the same expression for the electron

$$
E_{n}^{e( \pm)}= \pm \sqrt{m_{e}^{2}+k_{z}^{2}+2 n\left|Q_{e}\right| B}
$$

\subsection{Equation of State}

In this section we derive the equation of state (EOS). In order to do it in its general form we must include the temperature and then we can obtain the zero temperature case taking $T \rightarrow 0$ in all that we are about to derive.

In order to add the temperature in our half-classical and half-quantum theory we have to find the partition function as if it were all quantum:

$$
Z=\operatorname{Tr}\left\{\exp \left[-\left(\hat{H}-\mu_{e} \hat{N}_{e}-\sum_{f=u}^{d, s} \mu_{f} \hat{N}_{f}\right) / T\right]\right\}
$$

We can already guess the classical part.

$$
Z_{\text {classical }}=\exp \left[V\left\{-\frac{m_{G}{ }^{2}}{2} \alpha_{0}^{a} \alpha_{0}^{a}+b \phi_{0}{ }^{4}+\frac{B^{2}}{8 \pi}\right\} \frac{1}{T}\right]
$$

Nevertheless, the fermions are fully quantum and we to have use the formalism of finite temperature quantum field theory. All of this is done in Appendix B. The result for the full partition function is

$$
\begin{gathered}
Z=\exp \left[V\left\{-\frac{m_{G}{ }^{2}}{2} \alpha_{0}^{a} \alpha_{0}^{a}+b \phi_{0}{ }^{4}+\frac{B^{2}}{8 \pi}\right\} \frac{1}{T}\right] \\
\times \prod_{\vec{k}, s, n}\left\{1+\exp \left[-\left(\mathcal{E}_{n}^{e}-\mu_{e}\right) / T\right]\right\}\left\{1+\exp \left[-\left(\mathcal{E}_{n}^{e}+\mu_{e}\right) / T\right]\right\}
\end{gathered}
$$




$$
\times \prod_{f=u}^{d, s} \prod_{\vec{k}, s, n}\left\{1+\exp \left[-\left(\mathcal{E}_{n}^{f}-\nu_{f}\right) / T\right]\right\}\left\{1+\exp \left[-\left(\mathcal{E}_{n}^{f}+\nu_{f}\right) / T\right]\right\}
$$

where the energies are:

$$
\begin{aligned}
\mathcal{E}_{n}^{e} & =\sqrt{m_{e}^{2}+k_{z}^{2}+2 n\left|Q_{e}\right| B} \\
\mathcal{E}_{n}^{f} & =\sqrt{m_{f}^{2}+k_{z}^{2}+2 n\left|Q_{f}\right| B}
\end{aligned}
$$

$\mu_{e}$ is the electron chemical potential and we must redefine the $f \in\{u, d, s\}$ quark chemical potential as:

$$
\nu_{f} \equiv \mu_{f}+g_{h}\left(c_{i}^{\dagger} T_{i j}^{a} c_{j}\right) \alpha_{0}^{a}
$$

We see that the partition function exhibits the usual fermionic terms.

\section{Energy, Entropy and Pressure}

In order to find all of the usual thermodynamic quantities, we start by finding the thermodynamic potential and from that we go to other quantities very easily. This potential is given by

$$
\Omega=-T \ln Z \text {. }
$$

We want to express the thermodynamic quantities as a function of the usual fermion and anti-fermion distributions:

$$
d_{i} \equiv \frac{1}{1+e^{\left(\mathcal{E}_{i}-\nu_{i}\right) / T}} \quad \bar{d}_{i} \equiv \frac{1}{1+e^{\left(\mathcal{E}_{i}+\nu_{i}\right) / T}}
$$

We have:

$$
\begin{gathered}
\Omega=\left[-\frac{m_{G}{ }^{2}}{2} \alpha_{0}^{a} \alpha_{0}^{a}+b \phi_{0}{ }^{4}+\frac{B^{2}}{8 \pi}\right] V \\
-T \sum_{\vec{k}, s, n}\left\{\ln \left[1+e^{-\left(\mathcal{E}_{n}^{e}-\mu_{e}\right) / T}\right]+\ln \left[1+e^{-\left(\mathcal{E}_{n}^{e}+\mu_{e}\right) / T}\right]\right\} \\
-T \sum_{f=u}^{d, s} \sum_{\vec{k}, s, n}\left\{\ln \left[1+e^{-\left(\mathcal{E}_{n}^{f}-\nu_{f}\right) / T}\right]+\ln \left[1+e^{-\left(\mathcal{E}_{n}^{f}+\nu_{f}\right) / T}\right]\right\} .
\end{gathered}
$$


The pressure parallel to direction of the magnetic field is given by [33, $34,35]$

$$
p_{\|}=-\frac{\Omega}{V}
$$

i.e.

$$
\begin{gathered}
p_{\|}=\frac{m_{G}{ }^{2}}{2} \alpha_{0}^{a} \alpha_{0}^{a}-b \phi_{0}{ }^{4}-\frac{B^{2}}{8 \pi} \\
+\frac{T}{V} \sum_{\vec{k}, s, n}\left\{\ln \left[1+e^{-\left(\mathcal{E}_{n}^{e}-\mu_{e}\right) / T}\right]+\ln \left[1+e^{-\left(\mathcal{E}_{n}^{e}+\mu_{e}\right) / T}\right]\right\} \\
+\sum_{f=u}^{d, s} \frac{T}{V} \sum_{\vec{k}, s, n}\left\{\ln \left[1+e^{-\left(\mathcal{E}_{n}^{f}-\nu_{f}\right) / T}\right]+\ln \left[1+e^{-\left(\mathcal{E}_{n}^{f}+\nu_{f}\right) / T}\right]\right\}
\end{gathered}
$$

We must take a continuum limit on the sums over momentum. The Landau orbits are helices so their projection on the $z$ plane is circular. According to $[36,37,38,39]$ the discrete transverse momentum for the Landau levels is

$$
k_{\perp}^{2}=2 \nu|Q| B
$$

So an integral in momentum becomes

$$
\int d^{3} k \rightarrow \frac{|Q| B}{\left(2 \pi^{2}\right)} \sum_{n} \int_{-\infty}^{\infty} d k_{z}
$$

Since our sums are also over spin we will have a degeneracy factor that depends on the Landau level and our continuum limit is

$$
\frac{1}{V} \sum_{\vec{k}, s, n} \longrightarrow \frac{1}{(2 \pi)^{3}} \sum_{n} \gamma_{i}(n) \int d^{3} k=\frac{\left|Q_{i}\right| B}{(2 \pi)^{2}} \sum_{n} \gamma_{i}(n) \int_{-\infty}^{\infty} d k_{z}
$$

for the electron $\gamma_{e}(n)=\left(2-\delta_{n 0}\right)$ and for each quark $f$ we have $\gamma_{f}(n)=$ $3\left(2-\delta_{n 0}\right)$, where the factor " 3 " accounts for color, and the "2" for spin.

Applying all that to the parallel pressure we have

$$
p_{\|}=\frac{3 g_{h}^{2}}{2 N m_{G}^{2}} \rho^{2}-b \phi_{0}^{4}-\frac{B^{2}}{8 \pi}
$$




$$
\begin{array}{r}
-T \frac{\left|Q_{e}\right| B}{2 \pi^{2}} \sum_{n}\left(2-\delta_{n 0}\right) \int_{0}^{\infty} d k_{z}\left[\ln \left(1-d_{e}\right)+\ln \left(1-\bar{d}_{e}\right)\right] \\
-T \sum_{f=u}^{d, s} \frac{\left|Q_{f}\right| B}{2 \pi^{2}} \sum_{n} 3\left(2-\delta_{n 0}\right) \int_{0}^{\infty} d k_{z}\left[\ln \left(1-d_{f}\right)+\ln \left(1-\bar{d}_{f}\right)\right]
\end{array}
$$

where we have also used equation (2.36) to find $\rho^{a} \rho^{a}=\frac{3}{N} \rho^{2}$ where $\rho=$ $\rho_{u}+\rho_{d}+\rho_{s}$ and the gluon equation of motion (2.34) to express the pressure as a function of the quark density. A final manipulation of the integrals will give us the final expression:

$$
\begin{gathered}
p_{\|}=\frac{3 g_{h}{ }^{2}}{2 N m_{G}{ }^{2}} \rho^{2}-b \phi_{0}{ }^{4}-\frac{B^{2}}{8 \pi}+\frac{\left|Q_{e}\right| B}{2 \pi^{2}} \sum_{n}\left(2-\delta_{n 0}\right) \int_{0}^{\infty} d k_{z} \frac{k_{z}{ }^{2}}{\mathcal{E}_{n}^{e}}\left(d_{e}+\bar{d}_{e}\right) \\
+\sum_{f=u}^{d, s} \frac{\left|Q_{f}\right| B}{2 \pi^{2}} \sum_{n} 3\left(2-\delta_{n 0}\right) \int_{0}^{\infty} d k_{z} \frac{k_{z}{ }^{2}}{\mathcal{E}_{n}^{f}}\left(d_{f}+\bar{d}_{f}\right)
\end{gathered}
$$

The next several thermodynamic quantities we will need are obtained mostly by algebraic manipulation so most of the details will be omitted and some will be dealt with in Appendix B. The magnetization is

$$
M=-\frac{1}{V} \frac{\partial \Omega}{\partial B} \doteq \frac{\partial p_{\|}}{\partial B}
$$

so using (2.57) we have

$$
\begin{gathered}
M=-\frac{B}{4 \pi}-T \frac{\left|Q_{e}\right|}{2 \pi^{2}} \sum_{n}\left(2-\delta_{n 0}\right) \int_{0}^{\infty} d k_{z}\left[\ln \left(1-d_{e}\right)+\ln \left(1-\bar{d}_{e}\right)\right] \\
-T \sum_{f=u}^{d, s} \frac{\left|Q_{f}\right|}{2 \pi^{2}} \sum_{n} 3\left(2-\delta_{n 0}\right) \int_{0}^{\infty} d k_{z}\left[\ln \left(1-d_{f}\right)+\ln \left(1-\bar{d}_{f}\right)\right] \\
-\frac{\left|Q_{e}\right| B}{2 \pi^{2}} \sum_{n}\left(2-\delta_{n 0}\right) \int_{0}^{\infty} d k_{z}\left[\frac{d_{e} n\left|Q_{e}\right|}{\mathcal{E}_{n}^{e}}+\frac{\bar{d}_{e} n\left|Q_{e}\right|}{\mathcal{E}_{n}^{e}}\right] \\
-\sum_{f=u}^{d, s} \frac{\left|Q_{f}\right| B}{2 \pi^{2}} \sum_{n} 3\left(2-\delta_{n 0}\right) \int_{0}^{\infty} d k_{z}\left[\frac{d_{f} n\left|Q_{f}\right|}{\mathcal{E}_{n}^{f}}+\frac{\bar{d}_{f} n\left|Q_{f}\right|}{\mathcal{E}_{n}^{f}}\right]
\end{gathered}
$$

The perpendicular pressure, $p_{\perp}$, given by [33] 


$$
p_{\perp}=p_{\|}-M B
$$

is:

$$
\begin{aligned}
p_{\perp}= & \frac{3 g_{h}{ }^{2}}{2 N m_{G}{ }^{2}} \rho^{2}-b \phi_{0}{ }^{4}+\frac{B^{2}}{8 \pi}+\frac{\left|Q_{e}\right| B^{2}}{2 \pi^{2}} \sum_{n}\left(2-\delta_{n 0}\right) \int_{0}^{\infty} d k_{z}\left[\frac{d_{e} n\left|Q_{e}\right|}{\mathcal{E}_{n}^{e}}+\frac{\bar{d}_{e} n\left|Q_{e}\right|}{\mathcal{E}_{n}^{e}}\right] \\
& +\sum_{f=u}^{d, s} \frac{\left|Q_{f}\right| B^{2}}{2 \pi^{2}} \sum_{n} 3\left(2-\delta_{n 0}\right) \int_{0}^{\infty} d k_{z}\left[\frac{d_{f} n\left|Q_{f}\right|}{\mathcal{E}_{n}^{f}}+\frac{\bar{d}_{f} n\left|Q_{f}\right|}{\mathcal{E}_{n}^{f}}\right]
\end{aligned}
$$

The quark density is $\rho=\frac{\partial p_{\|}}{\partial \mu_{f}}$, so

$$
\rho=\sum_{f=u}^{d, s} \frac{\left|Q_{f}\right| B}{2 \pi^{2}} \sum_{n} 3\left(2-\delta_{n 0}\right) \int_{0}^{\infty} d k_{z}\left(d_{f}-\bar{d}_{f}\right)
$$

The entropy $s=\frac{\partial p_{\|}}{\partial T}$, i.e.

$$
\begin{gathered}
s=-\frac{\left|Q_{e}\right| B}{2 \pi^{2}} \sum_{n}\left(2-\delta_{n 0}\right) \int_{0}^{\infty} d k_{z}\left\{d_{e} \ln \left(d_{e}\right)+\left(1-d_{e}\right) \ln \left(1-d_{e}\right)\right. \\
\left.+\bar{d}_{e} \ln \left(\bar{d}_{e}\right)+\left(1-\bar{d}_{e}\right) \ln \left(1-\bar{d}_{e}\right)\right\} \\
-\sum_{f=u}^{d, s} \frac{\left|Q_{f}\right| B}{2 \pi^{2}} \sum_{n} 3\left(2-\delta_{n 0}\right) \int_{0}^{\infty} d k_{z}\left\{d_{f} \ln \left(d_{f}\right)+\left(1-d_{f}\right) \ln \left(1-d_{f}\right)\right. \\
\left.+\bar{d}_{f} \ln \left(\bar{d}_{f}\right)+\left(1-\bar{d}_{f}\right) \ln \left(1-\bar{d}_{f}\right)\right\}
\end{gathered}
$$

and finally the energy is calculated with the Gibbs relation

$$
\varepsilon=-p_{\|}+T s+\mu_{e} \rho_{e}+\sum_{f} \mu_{f} \rho_{f}
$$

which too requires algebraic manipulation and results in

$$
\varepsilon=\frac{3 g_{h}{ }^{2}}{2 N m_{G}^{2}} \rho^{2}+b \phi_{0}{ }^{4}+\frac{B^{2}}{8 \pi}+\frac{\left|Q_{e}\right| B}{2 \pi^{2}} \sum_{n}\left(2-\delta_{n 0}\right) \int_{0}^{\infty} d k_{z} \mathcal{E}_{n}^{e}\left(d_{e}+\bar{d}_{e}\right)
$$




$$
+\sum_{f=u}^{d, s} \frac{\left|Q_{f}\right| B}{2 \pi^{2}} \sum_{n} 3\left(2-\delta_{n 0}\right) \int_{0}^{\infty} d k_{z} \mathcal{E}_{n}^{f}\left(d_{f}+\bar{d}_{f}\right)
$$

\section{Zero Temperature Limit}

Here, we treat the interesting case of zero temperature, which was extensively discussed [40, 36, 37, 38, 39].

The main feature of this limit is that the distribution functions become

$$
d_{i}=\Theta\left(\nu_{i}-\mathcal{E}_{n}^{i}\right) \quad \text { and } \quad \bar{d}_{i}=0
$$

and also that [40]:

$$
\lim _{T \rightarrow 0} T \ln \left(1-d_{i}\right)=\left(\mathcal{E}_{n}^{i}-\nu_{i}\right) \quad \text { and } \quad \lim _{T \rightarrow 0} T \ln \left(1-\bar{d}_{i}\right)=0
$$

The quark density will thus become

$$
\begin{gathered}
\rho=\sum_{f=u}^{d, s} \frac{\left|Q_{f}\right| B}{(2 \pi)^{2}} \sum_{n=0}^{n_{\text {max }}^{f}} 3\left(2-\delta_{n 0}\right) \int_{-\infty}^{\infty} d k_{z} \Theta\left(\nu_{f}-\mathcal{E}_{n}^{f}\right) \\
=\sum_{f=u}^{d, s} \frac{\left|Q_{f}\right| B}{2 \pi^{2}} \sum_{n=0}^{n_{\max }^{f}} 3\left(2-\delta_{n 0}\right) \int_{0}^{k_{z, F}} d k_{z}=\sum_{f=u}^{d, s} \frac{\left|Q_{f}\right| B}{2 \pi^{2}} \sum_{n=0}^{n_{\text {max }}^{f}} 3\left(2-\delta_{n 0}\right) k_{z, F}^{f}(n)
\end{gathered}
$$

The distributions (2.64) imply that

$$
\sqrt{m_{f}^{2}+k_{z, F}^{f}{ }^{2}+2 n\left|Q_{f}\right| B}=\nu_{f} \Longrightarrow k_{z, F}^{f}=\sqrt{\nu_{f}^{2}-m_{f}^{2}-2 n\left|Q_{f}\right| B}
$$

which implies a maximum Landau number. Given that the momentum must be positive

$$
n \leq n_{\max }^{f}=\frac{\nu_{f}^{2}-m_{f}^{2}}{2\left|Q_{f}\right| B} .
$$

and the same goes for the electrons

$$
\rho_{e}=\frac{\left|Q_{e}\right| B}{2 \pi^{2}} \sum_{n=0}^{n_{\max }^{e}}\left(2-\delta_{n 0}\right) k_{z, F}^{e}(n)
$$




$$
\begin{gathered}
k_{z, F}^{e}=\sqrt{\mu_{e}^{2}-m_{e}^{2}-2 n\left|Q_{e}\right| B} \\
n \leq n_{\max }^{e}=\frac{\mu_{e}^{2}-m_{e}^{2}}{2\left|Q_{e}\right| B}
\end{gathered}
$$

Finally the energy and pressures will be

$$
\begin{aligned}
\varepsilon=\frac{3 g_{h}{ }^{2}}{2 N m_{G}{ }^{2}} \rho^{2}+b \phi_{0}{ }^{4}+\frac{B^{2}}{8 \pi}+\frac{\left|Q_{e}\right| B}{2 \pi^{2}} \sum_{n=0}^{n_{\max }^{e}}\left(2-\delta_{n 0}\right) \int_{0}^{k_{z, F}^{e}} d k_{z} \sqrt{m_{e}^{2}+k_{z}^{2}+2 n\left|Q_{e}\right| B} \\
\quad+\sum_{f=u}^{d, s} \frac{\left|Q_{f}\right| B}{2 \pi^{2}} \sum_{n=0}^{n_{\max }^{f}} 3\left(2-\delta_{n 0}\right) \int_{0}^{k_{z, F}^{f}} d k_{z} \sqrt{m_{f}^{2}+k_{z}^{2}+2 n\left|Q_{f}\right| B} \\
p_{\|}=\frac{3 g_{h}^{2}}{2 N m_{G}^{2}} \rho^{2}-b \phi_{0}^{4}-\frac{B^{2}}{8 \pi}+\frac{\left|Q_{e}\right| B}{2 \pi^{2}} \sum_{n=0}^{n_{\max }^{e}}\left(2-\delta_{n 0}\right) \int_{0}^{k_{z, F}^{e}} d k_{z} \frac{k_{z}{ }^{2}}{\sqrt{m_{e}^{2}+k_{z}^{2}+2 n\left|Q_{e}\right| B}} \\
\quad+\sum_{f=u}^{d, s} \frac{\left|Q_{f}\right| B}{2 \pi^{2}} \sum_{n=0}^{n_{\max }^{f}} 3\left(2-\delta_{n 0}\right) \int_{0}^{k_{z, F}^{f}} d k_{z} \frac{k_{z}{ }^{2}}{\sqrt{m_{f}^{2}+k_{z}^{2}+2 n\left|Q_{f}\right| B}}
\end{aligned}
$$

Defining the perpendicular pressure as 2.59 we have:

$$
\begin{aligned}
p_{\perp}= & \frac{3 g_{h}{ }^{2}}{2 N m_{G}{ }^{2}} \rho^{2}-b \phi_{0}{ }^{4}+\frac{B^{2}}{8 \pi}+\frac{\left|Q_{e}\right|^{2} B^{2}}{2 \pi^{2}} \sum_{n=0}^{n_{\max }^{e}}\left(2-\delta_{n 0}\right) n \int_{0}^{k_{z, F}^{e}} \frac{d k_{z}}{\sqrt{m_{e}^{2}+k_{z}^{2}+2 n\left|Q_{e}\right| B}} \\
& +\sum_{f=u}^{d, s} \frac{\left|Q_{f}\right|^{2} B^{2}}{2 \pi^{2}} \sum_{n=0}^{n_{\max }^{f}} 3\left(2-\delta_{n 0}\right) n \int_{0}^{k_{z, F}^{f}} \frac{d k_{z}}{\sqrt{m_{f}^{2}+k_{z}^{2}+2 n\left|Q_{f}\right| B}}
\end{aligned}
$$

These equations for pressure, entropy and energy are what define the EOS. In the following chapter we will apply this EOS to the study of quark stars. 


\section{Chapter 3}

\section{Stellar Structure with a Magnetic Equation Of State}

One of the possible applications of the equations of state described in Chapter 2 is the study of the structure of compact stars. In 1939 both Tolman, Oppenheimer and Volkoff derived a differential equation based only on Einstein gravitational theory for the structure of compact astrophysical objects. The final equation receives as an input the EOS in the form $\epsilon=\epsilon(p)$ and returns a curve on the Mass $\times$ Radius plane describing possible stars with those values of mass and radius. The derivation is presented in Appendix A.

The TOV equations read:

$$
\frac{d P(r)}{d r}=-\frac{1}{r^{2}}(\epsilon(r)+P(r))\left(M(r)+4 \pi r^{3} P(r)\right)\left(1-\frac{2 M(r)}{r}\right)^{-1}
$$

where $G$, the gravitational constant and $c$, the speed of light, are both set to 1 , and the mass is given by the continuity equation.

$$
\frac{d M(r)}{d r}=4 \pi r^{2} \epsilon(r)
$$

The two main assumptions taken on deriving this equation, as shown in the Appendix A, are isotropy and spherical symmetry. None of those two characteristics are present in our equations of state, particularly in $(2.71,2.72,2.73)$ the presence of a constant magnetic field in the $\hat{z}$ direction breaks explicitly both the isotropy and the spherical symmetry. However, as we can see in 
figure 3.1 the difference between the parallel and perpendicular pressures is roughly zero until a certain critical point where it starts to grow very fast. Given this behavior we decided [1] to utilize the TOV equations (TolmanOppenheimer-Volkof) and to present our results and predictions for the stellar structure using both the parallel and perpendicular pressure respectively as an inner and outer limit. That assumption is well explained in figure 3.2.

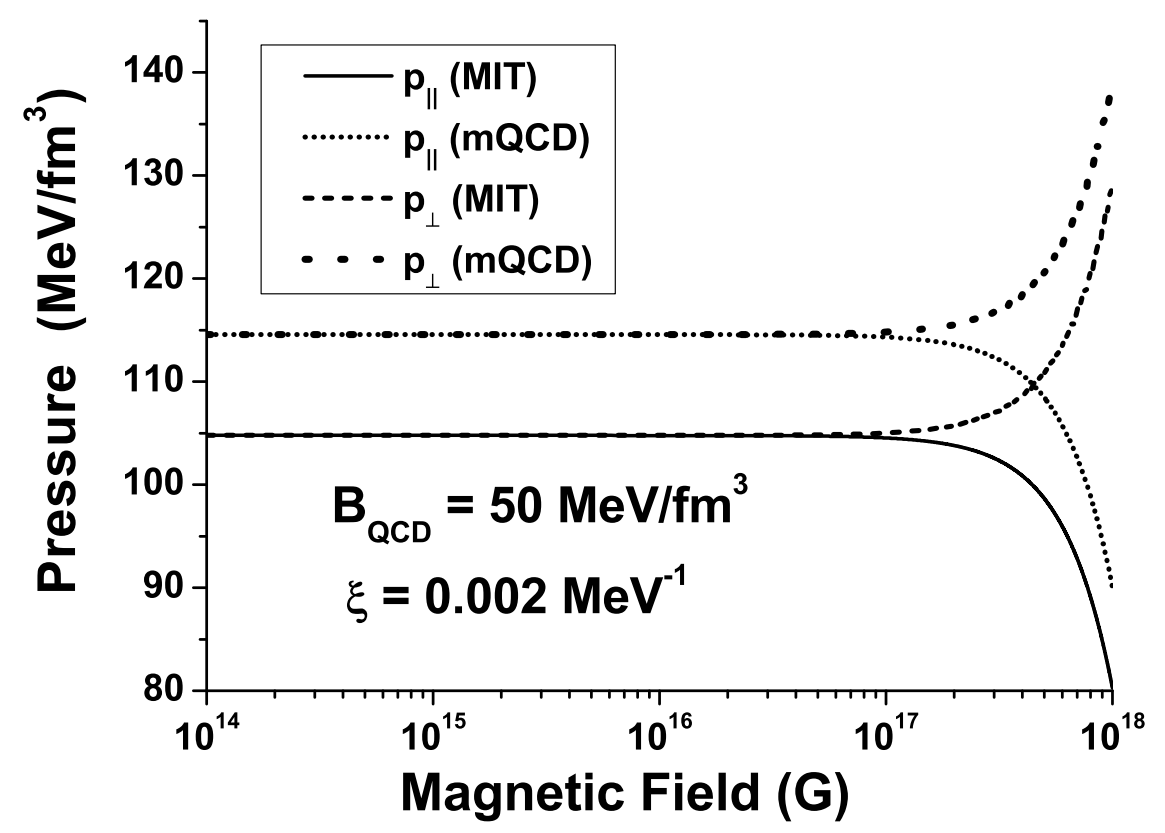

FIGURE 3.1: The splitting between the paralell and perpendicular pressures for $\mathrm{MQCD}$ and for the MIT bag model as a function of the magnetic field, where $\xi \doteq g_{h} / m_{G}$

The difference between both results is evaluated as a theoretical error on the Mass $\times$ Radius diagram. As we will show, the maximum magnetic field for this approach to be valid is $5 \times 10^{17} G$. For fields higher than this value the difference between the parallel and perpendicular pressures is much too large and grows quickly with increasing magnetic fields, see Figure 3.1

Also, note that in figure 3.1 the mQCD EOS is always stiffer than the MIT bag model (the MIT bag model and mQCD coincide for $\xi \doteq g_{h} / m_{G}=0$, see equations $2.71,2.72,2.73)$. The pressures of both EOSs split in exactly the 


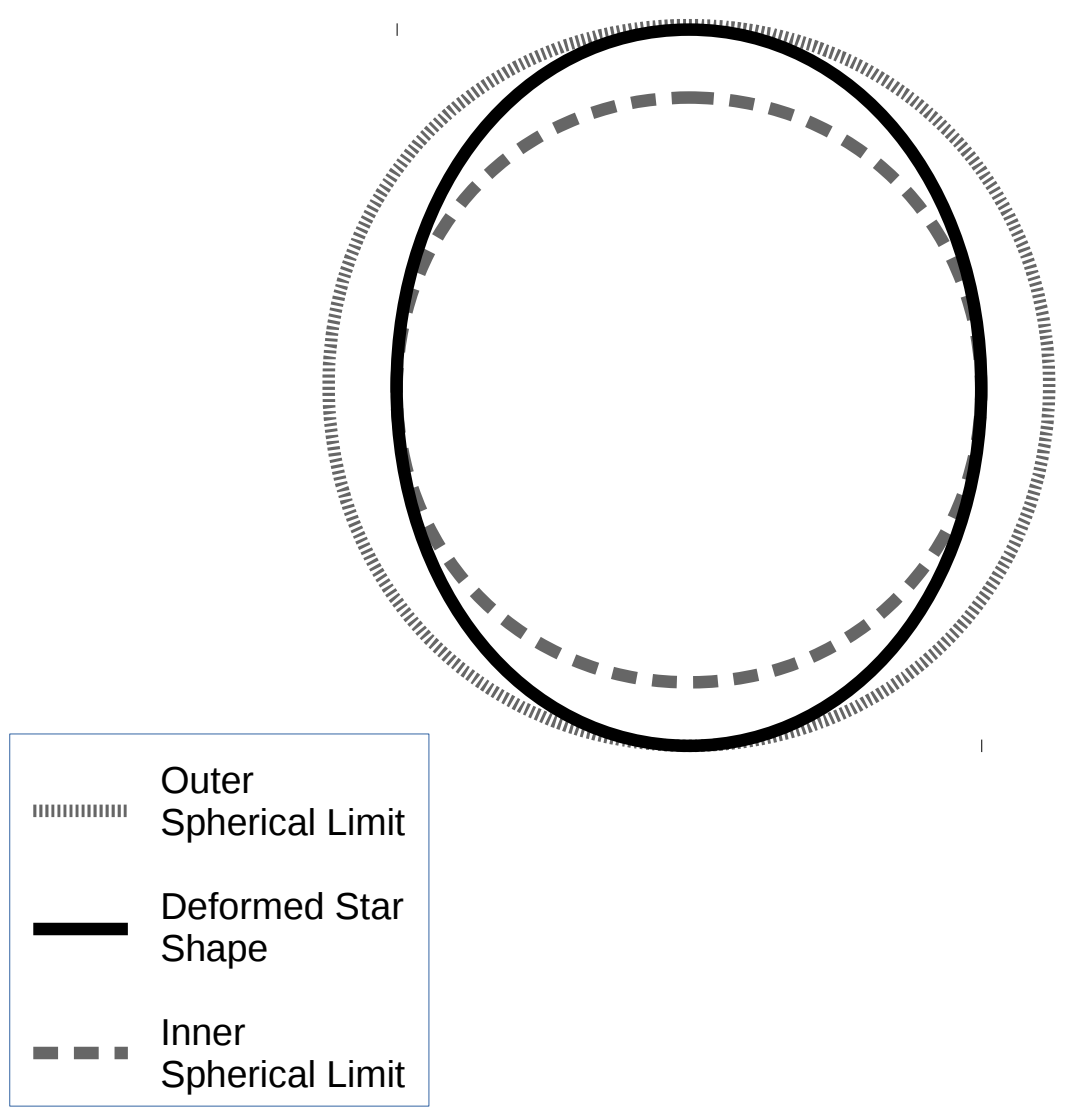

FIGURE 3.2: Inner and Outer spherical limits

same point, therefore, everything that can be said of the mQCD EOS regarding the applicability of the TOV can also be said of the MIT bag model. That makes it very easy to compare both equations of state.

\subsection{Stability Conditions}

As already discussed in the introduction, in order to apply the model to the study of compact stars we have to make sure some conditions hold. A realistic model of a compact stars would have to conserve the baryonic density, be neutrally charged, allow beta decay to be in chemical equilibrium 
and make sure that the physical parameters are set to values for which the plasma would not hadronize.

Here we model only pure quark stars but it is possible to model neutron stars as a hybrid system where its core is made of a cold QGP and its outer layer is made of hadrons. For some studies on hybrid stars see [41, 42, 43].

As explained in the Introduction, the weak processes involving quarks

$$
\begin{gathered}
u+e^{-} \rightarrow d+\nu_{e} \\
u+e^{-} \rightarrow s+\nu_{e} \\
u+s \rightarrow d+u \\
s \rightarrow u+\nu_{e}+\bar{\nu}_{e} \\
d \rightarrow u+\nu_{e}+\bar{\nu}_{e}
\end{gathered}
$$

will impose the conditions

$$
\nu_{d}=\nu_{s}=\nu_{u}+\mu_{e}
$$

The conservation of baryonic density $\left(\rho_{B}\right)$ and charge neutrality respectively yield the conditions

$$
\begin{array}{r}
3 \rho_{B}=\rho_{u}+\rho_{d}+\rho_{s} \\
\frac{2}{3} \rho_{u}=\frac{1}{3} \rho_{d}+\frac{1}{3} \rho_{s}+\rho_{e} .
\end{array}
$$

At last we must impose, as demonstrated in [6], that the energy per baryonic number density be lower than the energy necessary for the system to start producing hadrons on its reactions and that the energy per baryonic density of the system with only two quark flavors ( $u$ and $d$ ) be higher than the minimum energy required to unbind the quarks $u$ and $d$. I.e. the lower limit guarantees that the energy is enough to dissolve the nucleons and the upper limit guarantees that the system will not react and produce hadrons. Therefore, we make sure that the system is in a QGP state.

These restrictions translate to 


$$
\left.\frac{\varepsilon}{\rho_{B}}\right|_{(3 \text {-flavor })} \leq 934 \mathrm{MeV} \leq\left.\frac{\varepsilon}{\rho_{B}}\right|_{(2 \text {-flavor })}
$$

\section{Stability Window}

Conditions 3.8 to 3.9 are just a matter of choosing the right set of values for the chemical potentials. We need values for which the condition 3.8 and also (once the densities are calculated using 2.66 and 2.68) the conditions 3.10 and 3.9 are satisfied.

Satisfying condition 3.11 is not that simple. Once the chemical potentials and densities are set, the energy will have to be inside this stability interval. The energy is

$$
\begin{gathered}
\varepsilon=\frac{3 g_{h}{ }^{2}}{2 N m_{G}^{2}} \rho^{2}+b \phi_{0}{ }^{4}+\frac{B^{2}}{8 \pi}+\frac{\left|Q_{e}\right| B}{2 \pi^{2}} \sum_{n=0}^{n_{\max }^{e}}\left(2-\delta_{n 0}\right) \int_{0}^{k_{z, F}^{e}} d k_{z} \sqrt{m_{e}^{2}+k_{z}^{2}+2 n\left|Q_{e}\right| B} \\
+\sum_{f=u}^{d, s} \frac{\left|Q_{f}\right| B}{2 \pi^{2}} \sum_{n=0}^{n_{\max }^{f}} 3\left(2-\delta_{n 0}\right) \int_{0}^{k_{z, F}^{f}} d k_{z} \sqrt{m_{f}^{2}+k_{z}^{2}+2 n\left|Q_{f}\right| B}
\end{gathered}
$$

which for simplicity we will call

$$
\varepsilon=\frac{3 \xi^{2}}{2 N} \rho^{2}+B_{Q C D}+\frac{B^{2}}{8 \pi}+I_{\text {fermions }}
$$

Where $I_{\text {fermions }}$ denotes the integrals involving fermions, we identify the 4-dimensional gluon condensate as a Bag-like term $b \phi_{0}{ }^{4} \rightarrow B_{Q C D}$, and rename $\xi=\frac{g_{h}}{m_{G}}$.

In this form we see that once the chemical potentials are fixed, so are the densities and two parameters remain to be chosen. The so called $B_{Q C D}$ and $\xi$. Using conditions 3.11 we find a domain where these values are such that a strange star is stable. This is what we will call stability sindow (see, for instance, figure 3.3).

In [1], we chose some particular values of densities, relative to the nuclear density, $\frac{\rho_{B}}{\rho_{0}}$ and plotted two of this windows in figure 3.3 always using quark masses as $m_{u}=5 \mathrm{MeV}, m_{d}=7 \mathrm{MeV}$, and $m_{s}=150 \mathrm{MeV}$.

Therefore, in order to have a stable set of parameters for the star we must choose $B_{Q C D}$ and $\xi$ inside the region delimited by these curves. However, 


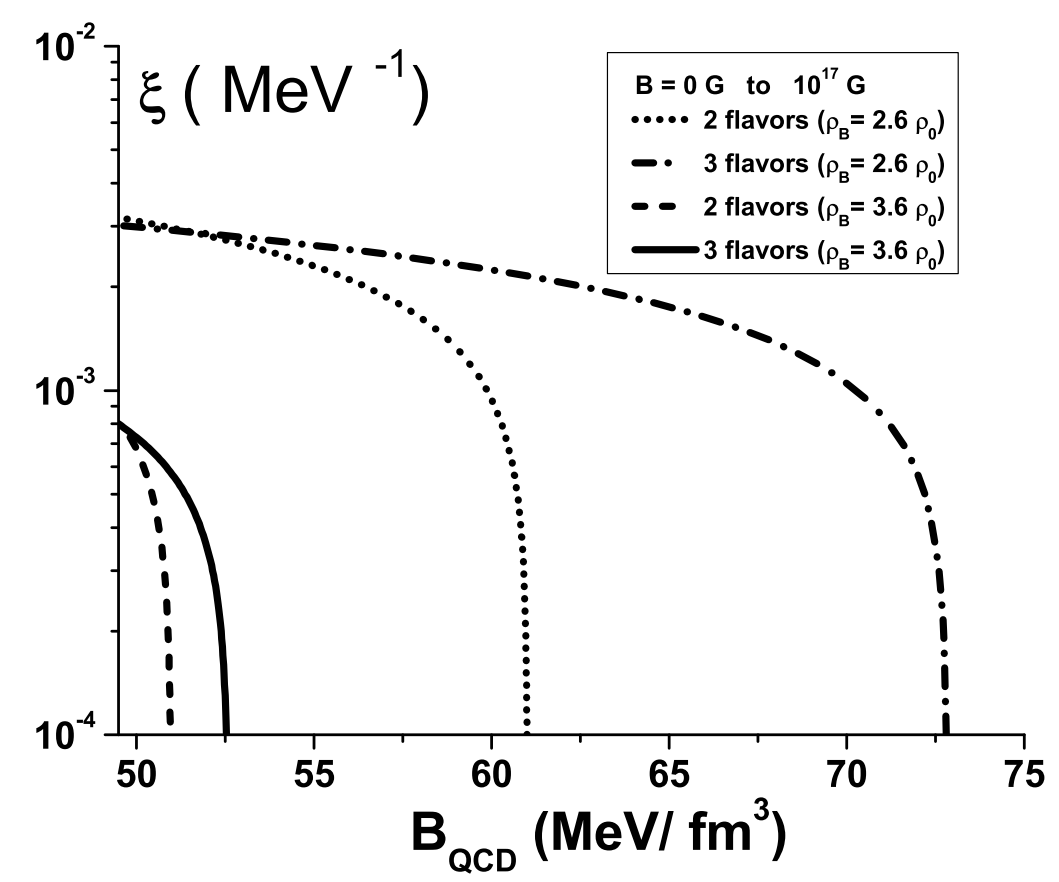

FIGURE 3.3: Stability windows defined by the conditions (1.3) to (1.4).

finding the window for the other parameters of the system, such as the density and the magnetic field, is hardly as simple. Eventually a certain set of parameters will render the window completely negative and therefore totally excluding the possibility of a stable star.

On figure 3.4 we show separately the regions where it is possible to find chemically balanced stars (light shaded), the region where the stability window still presents positive solutions for a pair $\left(B_{Q C D}, \xi\right)$, i.e. $3.7<\rho_{B} / \rho_{0}<$ 1.5 , and their intersection is the region where we can find perfectly stable quark stars (dark shaded). This figure is exactly the same for the MIT bag model EOS (with minor differences of negligible orders of magnitude) and so is all of this study regarding the stability.

In what follows, all values were chosen inside the intersection region.

Note also that this part of the study, regarding the stability, does not concern the functional form of the parallel and perpendicular pressures. Although we have restrained our study to stars with magnetic fields lower than $5 \times 10^{17} \mathrm{G}$ and therefore we did not perform any simulations with larger 


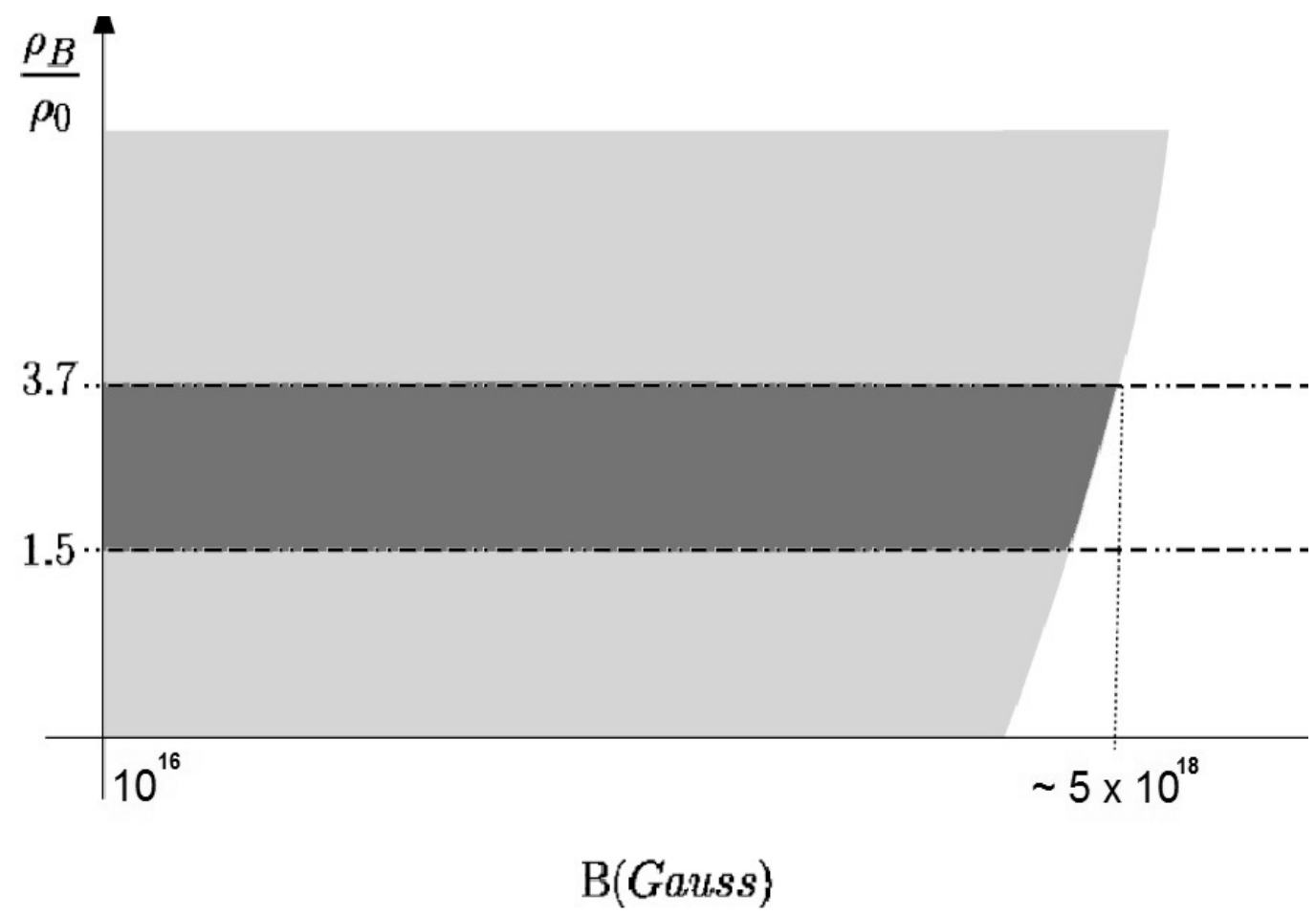

FIGURE 3.4: Stability diagram: baryon density ratio as function of the magnetic field. The points in the light gray area satisfy the conditions (1.3), (1.1) and (3.9). Points in the dark gray area satisfy also the condition (1.4).

values, the study regarding their stability is valid for every value of the magnetic field. The prediction that we will not find any stable stars with fields higher than $5 \times 10^{18} G$ is perfectly valid.

\subsection{Mass-Radius Results}

As interesting as it can be investigating the darker corners of the stability conditions for the star, we are mainly interested in the consequences of this particular EOS to the structure of these stars.

We solved numerically the TOV equations, using only stable parameters, and using pressure and energy given by the two EOSs, the MIT bag model and $\mathrm{mQCD}$. That allows us to obtain the Mass $\times$ Radius diagrams for the models and see if they match the available experimental data. 
Integrating the TOV equations from inside to outside, the initial conditions for the center of the star $(r=0)$ are always given by $M(r=0)=0$, $P(r=0)=p$ where $p$ is the calculated pressure using the appropriate EOS and $\epsilon(r=0)=\epsilon$ where $\epsilon$ is also calculated using the EOS.

We then stop the integration when the pressure reaches zero, indicating the surface of the star. The total mass and the radius $R$ where $P(R)=0$ are respectively the mass and radius of the star. That calculation gives us one single value of mass and one single value of radius. However, in order to chech if the result is reliable, we perform the same procedure for an interval of several values of pressure centered in $p$, i.e. we solve for every pressure in $[p-\delta, p+\delta]$. If the solution presents the usual spirally curved shape (see figure 1.7 for SQM) that means that the solution is stable (or more specifically, its fundamental radial mode of oscillation is stable, see [7]) and the maximum mass of this curve is the mass limit for a star with these parameters.

The Figure 3.5 shows the different Mass $\times$ Radius diagrams for $m Q C D$ and the MIT bag model with magnetic field values of zero and $5 \times 10^{16} \mathrm{G}$. $\mathrm{Up}$ to this values of magnetic fields the equations of state of quark matter suffer little or no change. They are insensitive to fields lower than this value.

However, we can see that $\mathrm{QQCD}$ already presents higher values of allowed masses.

Nonetheless, in Figures 3.6(a) and 3.6(b) the influence of the magnetic field is much more pronounced. We can see the behavior of the system with the mQCD parameters, the $\xi$ and $\mathcal{B}_{Q C D}$.

Increasing $\mathcal{B}_{Q C D}$, which is an external pressure that the star feels from the vacuum, always diminishes the maximum values of mass and radius. Increasing $\xi$, however, increases the strength of the EOS and therefore yields larger values of mass and radius.

Finally, we do this for several values of magnetic field and plot the maximum mass as a function of the magnetic field in figure 3.7. Limiting our analysis to fields of $5 \times 10^{17} G$ we see that the difference between the upper and lower limit results are of around $10 \%$ of the value without magnetic field. Therefore, we conclude that up to that value of magnetic field the TOV equation can be used as a simplistic approximation if we take into account this theoretical error. 


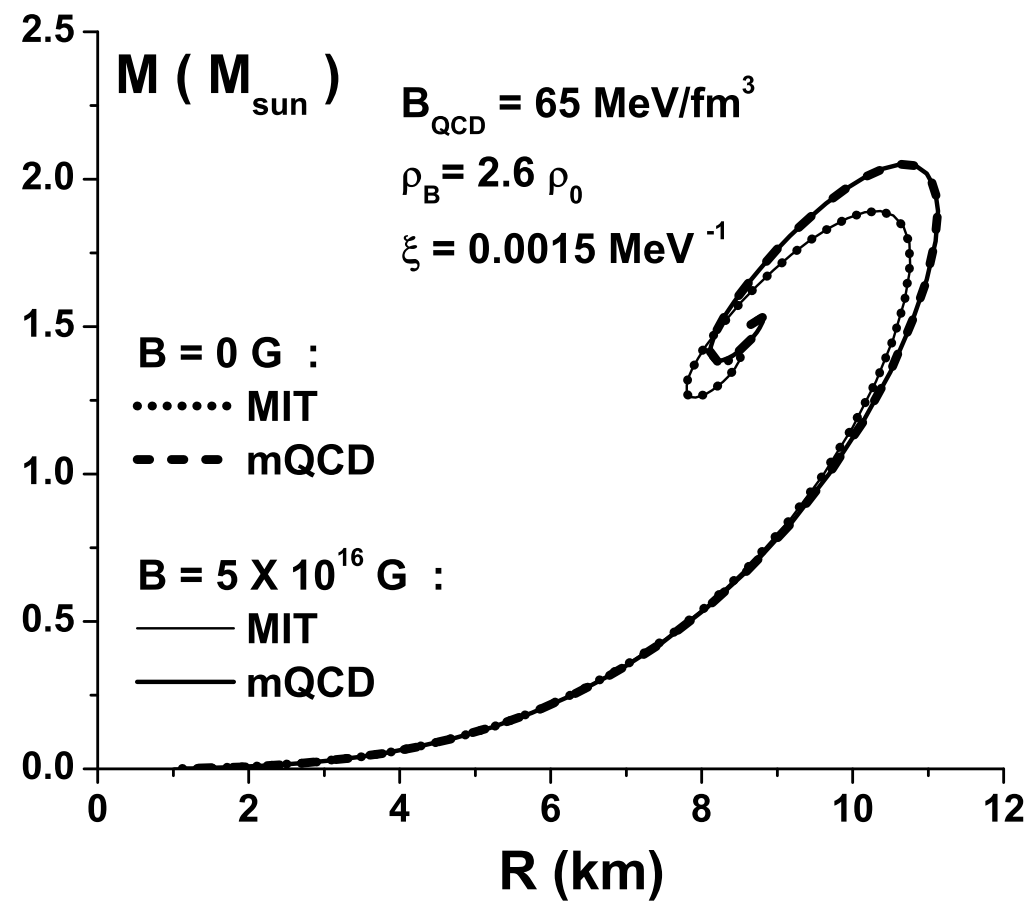

FIGURE 3.5: Mass-radius diagrams. Two values of the magnetic fields with $\mathcal{B}_{Q C D}$ and $\xi$ allowed by the stability conditions at central density $\rho_{B}=2.6 \rho_{0}$. The largest masses are 2.05 (mQCD) and 1.89 (MIT). In these cases $p_{\|}=p_{\perp}$ which permits the use of TOV.

We also see that, as expected, the MQCD EOS is always stiffer than the MIT bag model and yields larger masses. 


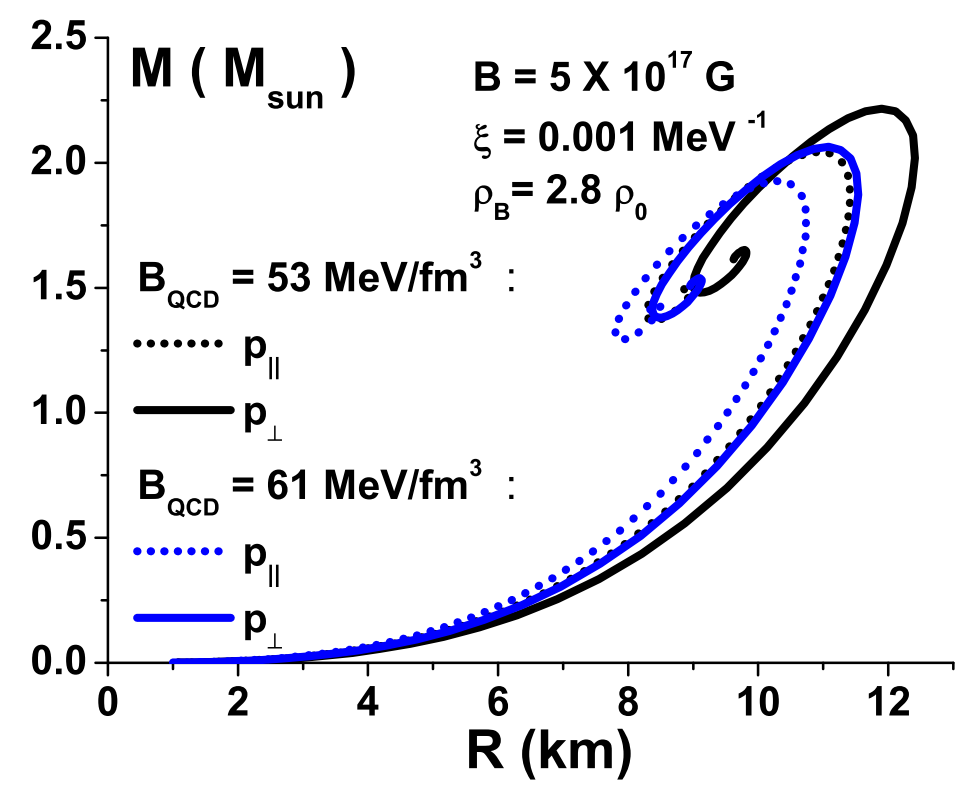

(a)

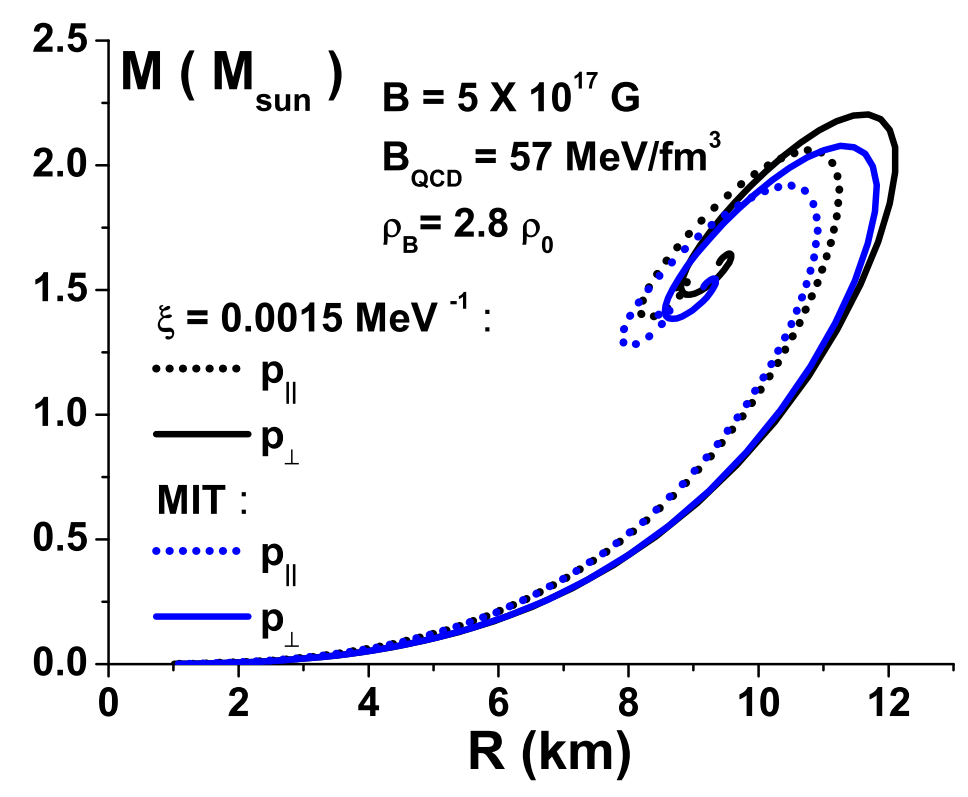

(b)

FIGURE 3.6: Mass-radius diagram for a fixed value of the magnetic field and baryon density with.

(a) Fixing $\xi$ and varying $\mathcal{B}_{Q C D}$ : For $\mathcal{B}_{Q C D}=53 \mathrm{MeV} / \mathrm{fm}^{3}$ the higher masses are 2.22 and 2.04 (dotted lines). Whereas for $\mathcal{B}_{Q C D}=61 \mathrm{MeV} / \mathrm{fm}^{3}$ masses are of up to 2.06 and 1.93 (solid lines).

(b) Fixing $\mathcal{B}_{Q C D}$ and varying $\xi$ : For $\xi=0.0015 \mathrm{MeV}^{-1}$ the largest masses are 2.20 and 2.06(dotted lines). Whereas for $\operatorname{MIT}\left(\xi=0 \mathrm{MeV}^{-1}\right)$ the largest masses are 2.08 and 1.92 (solid lines). 


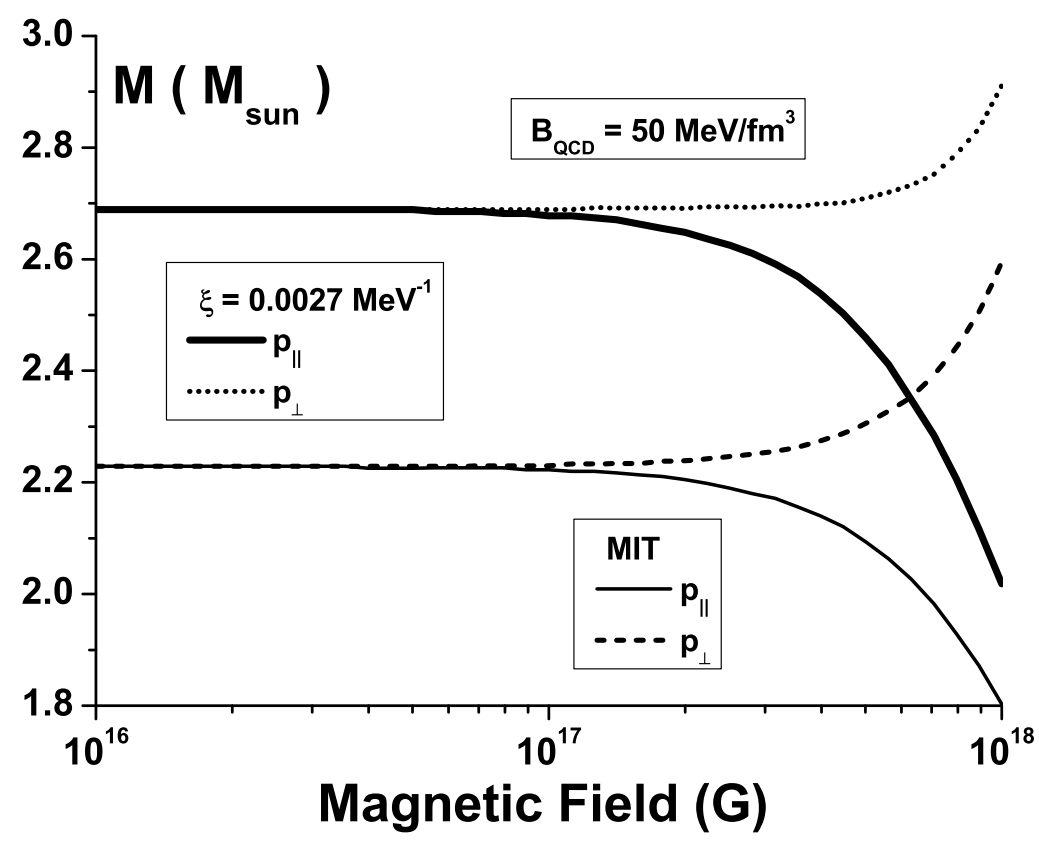

FIGURE 3.7: Effects of the splitting of parallel and perpendicular pressures on the maximum masses calculated with the chemical potentials obeying the stability conditions (1.3), (1.1) and (3.9)), given by $\nu_{u}=300 \mathrm{MeV}, \nu_{d}=\nu_{s}=316.5 \mathrm{MeV}$ and $\mu_{e}=16.5 \mathrm{MeV}$. 



\section{Chapter 4}

\section{Conclusion}

In Chapter 2 we have derived a new form of the mQCD EOS for very high densities and low temperatures, where the quark matter suposed to exist inside neutron stars is described, directly from the QCD Lagrangian including the influence of the magnetic field. Having performed those calculations with these approximations, we were able to recover the MIT bag model as a theoretical limit when $\xi \rightarrow 0$ (and also the Stefan-Boltzman limit for $\left.\mathcal{B}_{Q C D}=\xi \rightarrow 0\right)$.

Furthermore, we performed simulations and determine that this EOS is compatible with the experimental results given in $[10,9]$. Stars with magnetic fields of $B=5 \times 10^{17} \mathrm{G}$ can support masses of up to $2.22 M_{\odot}$ and $2.20 M_{\odot}$ for different stable values of $\xi$ and $\mathcal{B}_{Q C D}$ and radius of $12 \mathrm{~km}$ at most which is compatible with theoretical expectations.

Regarding the stability of the star we determine well defined values and ranges of parameters guaranteeing the chemical equilibrium and stability for $\mathrm{mQCD}$ and for the MIT bag model. We have also observed that by increasing the magnitude of the magnetic field the stability window starts to shrink and vanishes completely at $B=5 \times 10^{18} \mathrm{G}$. Since this calculation is exactly the same for the MIT bag model we have determined that for both EOSs, no stable star of pure quark matter can exist with such values of magnetic field. The same goes for any EOS that has the same expression for densities and that has values of energy density comparable to those of the MIT bag model. The functional form of parallel and perpendicular pressures changes nothing to this result.

We calculated the Mass $\times$ Radius diagrams for a range of values of magnetic fields (figure 3.7). In this particular model the different mass curves, one calculated with the parallel pressure and the other with perpendicular 
pressure, start to split around $B=10^{17} G$ and this difference increases up to the value that we decide that this approximation starts to lose its validity, $B=5 \times 10^{17} G$, where the difference between the curves is $10 \%$ of the value without magnetic field, where they agree completely. The same goes for the MIT bag model.

Although it is still unclear what is the full effect of the magnetic field, one thing can be said for sure, the reliability of every study made with this type of models for strange quark matter that did not account for the influence of the magnetic field is now possible to estimate. For fields below the threshold of our study $B<10^{18} G$ the theoretical error is of $10 \%$ at most.

Summarizing: the presented equations of state are completely insensitive to low values of the magnetic field, $B<10^{16} \mathrm{G}$. For fields with magnitude such that $10^{16} G<B<5 \times 10^{17} G$ the theoretical error is estimated to be $10 \%$ at most. The inclusion of the magnetic field, in the mQCD, does not change the fact that the model is compatible with recent experimental observations of pulsar masses, i.e. $M_{M A X}>2.22 M_{\odot}$ for every calculation. And no stable strange star can be found, both in chemical equilibrium and fulfilling the condition 3.11 of stability, with fields larger than $B \approx 5 \times 10^{18} G$, assuming the uniform magnetic field. 


\section{Appendix A}

\section{Derivation of the TOV equation}

To model a compact star with General Relativity (GR) we have to make some theoretical assumptions. Here we assume that the star is spherically symmetrical, isotropic, and composed of an ideal fluid. Those are all sufficiently reasonable approximations to model neutron stars. Of course that if one wishes to account for the deforming effects of rotation and magnetic field one will have to perform much more delicate calculations leading to a more realistic mode.

Assuming that the system is spherically symmetric, the metric of spacetime is the usual Schwarzschild metric (with $c=G=1$ ):

$$
d s^{2}=e^{\nu(r)} d t^{2}-e^{\lambda(r)} d r^{2}-r^{2} d \theta^{2}-r^{2} \sin \theta d \phi^{2}
$$

Where $\nu$ and $\lambda$ are metric functions to be determined.

And assuming also that the system is composed of an isotropic pressured ideal fluid, i.e.

$$
T^{00}=\epsilon(r)
$$

and

$$
T^{i j}=-P(r) \delta^{i j}
$$

where $\epsilon$ is the energy density and $P$ is the pressure.

We now solve the Einstein field equations: 


$$
8 \pi T^{\mu \nu}=G^{\mu \nu} \quad \text { where } G^{\mu \nu} \equiv R^{\mu \nu-\frac{1}{2} R g^{\mu \nu}}
$$

From the Schwarzschild metric we can determine the Ricci tensor $R^{\mu \nu}$ and the metric elements $g^{\mu \nu}$.

Considering the first element of the equation we have:

$$
8 \pi \epsilon(r) e^{\nu(r)}=\frac{e^{\nu(r)}}{r^{2}}\left(1-\frac{d}{d r} r e^{-\lambda(r)}\right)
$$

Which integrated gives

$$
e^{-\lambda(r)}=1-\frac{2 M(r)}{r}
$$

And therefore determines the first metric function as a function of a physical parameter, the mass.

Now consider, for instance, the element $(1,1)$ :

$$
-8 \pi P(r) e^{\lambda(r)}=\frac{-r \dot{\nu}(r)+e^{\lambda(r)}-1}{r^{2}}
$$

which using equation A.6 simplifies to

$$
\frac{d \nu(r)}{d r}=\frac{1}{r}\left(\frac{2 M(r)}{r}+8 \pi r^{2} P(r)\right)\left(1-\frac{2 M(r)}{r}\right)^{-1}
$$

Using the continuity equation $\nabla_{\mu} T_{\nu}^{\mu}=0$, a static and isotropic system (vanishing all time and angular derivatives of $\epsilon$ and $P$ ) we have

$$
\nabla_{\mu} T_{1}^{\mu}=-\frac{d P(r)}{d r}-\frac{1}{2}(P+\epsilon) \frac{d \nu(r)}{d r}=0
$$


Putting equations A.8 and A.9 together we have the so called TolmanOppenheimer-Volkof equations (TOV)

$$
\frac{d P(r)}{d r}=-\frac{1}{r^{2}}(\epsilon(r)+P(r))\left(M(r)+4 \pi r^{3} P(r)\right)\left(1-\frac{2 M(r)}{r}\right)^{-1}
$$

And the mass is given by the continuity equation

$$
\frac{d M(r)}{d r}=4 \pi r^{2} \epsilon(r)
$$





\section{Appendix B}

\section{Finite Temperature Fermion Field}

In finite temperature we will have both particles and antiparticles due to Fermi's Sea. The calculations below are done as on the references [40, 31, 36].

We start by the definition of the partition function:

$$
Z=\operatorname{Tr}\left\{\exp \left[-\left(\hat{H}-\mu_{e} \hat{N}_{e}-\sum_{f=u}^{d, s} \mu_{f} \hat{N}_{f}\right) / T\right]\right\}
$$

in which $\hat{H}$ is the Hamiltonian and the $\hat{N}_{\text {type }}$ operators are the number operators for a certain particle type.

Opening the partition function:

$$
\begin{gathered}
Z=\operatorname{Tr}\left\{\exp \left[-\left(\hat{H}-\mu_{e} \hat{N}_{e}-\sum_{f=u}^{d, s} \mu_{f} \hat{N}_{f}\right) / T\right]\right\}= \\
\sum_{n_{1}, n_{2}, \ldots, n_{\infty}}\left\langle n_{1}, n_{2}, \ldots, n_{\infty}\left|\exp \left[-\left(\hat{H}-\mu_{e} \hat{N}_{e}-\sum_{f=u}^{d, s} \mu_{f} \hat{N}_{f}\right) / T\right]\right| n_{1}, n_{2}, \ldots, n_{\infty}\right\rangle
\end{gathered}
$$

Since the states are eigenstates of this operators we can just use their eigenvalues

$$
\begin{gathered}
Z=\sum_{n_{1}, n_{2}, \ldots, n_{\infty}}\left\langle n_{1}, n_{2}, \ldots, n_{\infty}\right| \exp \left[\left(-\sum_{i=1}^{\infty} E_{i} n_{i}+\mu_{e} \sum_{i=1}^{\infty} n_{i}\right.\right. \\
\left.\left.+\sum_{f=u}^{d, s} \mu_{f} \sum_{i=1}^{\infty} n_{i}\right) / T\right]\left|n_{1}, n_{2}, \ldots, n_{\infty}\right\rangle \\
=\sum_{n_{1}}\left\langle n_{1}\left|\exp \left[\left(-E_{1} n_{1}+\mu_{e} n_{1}+\sum_{f=u}^{d, s} \mu_{f} n_{1}\right) / T\right]\right| n_{1}\right\rangle \times
\end{gathered}
$$




$$
\begin{gathered}
\times \sum_{n_{2}}\left\langle n_{2}\left|\exp \left[\left(-E_{2} n_{2}+\mu_{e} n_{2}+\sum_{f=u}^{d, s} \mu_{f} n_{2}\right) / T\right]\right| n_{2}\right\rangle \ldots \\
\cdots \sum_{n_{\infty}}\left\langle n_{\infty}\left|\exp \left[\left(-E_{\infty} n_{\infty}+\mu_{e} n_{\infty}+\sum_{f=u}^{d, s} \mu_{f} n_{\infty}\right) / T\right]\right| n_{\infty}\right\rangle \\
=\prod_{i=1}^{\infty} \operatorname{Tr}_{i}\left\{\exp \left[\left(\mu_{e}+\sum_{f=u}^{d, s} \mu_{f}-E_{i}\right) n_{i} / T\right]\right\}
\end{gathered}
$$

Fermionic fields can have only the occupation numbers 0 and 1 as a consequence of the anti-commutation relations, therefore

$$
\begin{aligned}
Z & =\prod_{i=1}^{\infty} \sum_{n=0}^{1}\left\{\exp \left[\left(\mu_{e}+\sum_{f=u}^{d, s} \mu_{f}-E_{i}\right) n / T\right]\right\} \\
& =\prod_{i=1}^{\infty}\left\{1+\exp \left[\left(\mu_{e}+\sum_{f=u}^{d, s} \mu_{f}-E_{i}\right) / T\right]\right\}
\end{aligned}
$$

To go on we must calculate

$\hat{N}_{e}, \hat{N}_{f}$ and $\hat{H}$. Starting with $\hat{N}_{e}$ :

$$
\hat{N}_{e}=\hat{\mathcal{N}}_{e}-\left\langle 0\left|\hat{\mathcal{N}}_{e}\right| 0\right\rangle
$$

where:

$$
\begin{gathered}
\langle 0|(\text { expression with operators })|0\rangle \equiv \\
\equiv\langle 0|(\text { terms containing operators of the expression })|0\rangle
\end{gathered}
$$

as in [40]. Analogously for the quarks :

$$
\hat{N}_{f}=\hat{\mathcal{N}}_{f}-\left\langle 0\left|\hat{\mathcal{N}}_{f}\right| 0\right\rangle
$$

where $f=u, d, s$. The hamiltonian $\hat{H}$ is:

$$
\hat{H}=\hat{\mathcal{H}}-\langle 0|\hat{\mathcal{H}}| 0\rangle
$$

In (B.5), (B.6) e (B.7) we have to compute the principal operators:

$$
\hat{\mathcal{N}}_{e}=\int d^{3} x \bar{\psi}^{e} \gamma^{0} \psi^{e}
$$




$$
\hat{\mathcal{N}}_{f}=\int d^{3} x \bar{\psi}^{f} \gamma^{0} \psi^{f}
$$

and

$$
\hat{\mathcal{H}}=\int d^{3} x\left\{\sum_{f=u}^{d, s} \frac{\partial \mathcal{L}_{0}}{\partial\left(\partial_{0} \psi^{f}\right)}\left(\partial^{0} \psi^{f}\right)+\frac{\partial \mathcal{L}_{0}}{\partial\left(\partial_{0} \psi^{e}\right)}\left(\partial^{0} \psi^{e}\right)-g^{00} \mathcal{L}_{0}\right\}
$$

The fermionic fields in terms of operators are the following. For quarks:

$$
\begin{aligned}
\psi_{i}^{f}= & c_{i} \frac{1}{\sqrt{V}} \sum_{\vec{k}, s, n}\left[A_{\vec{k}, s, n}^{f} \mathcal{U}^{f}(\vec{k}, s, n) e^{i \vec{k} \cdot \vec{x}-i E_{n}^{f(+)} t}\right. \\
& \left.+B^{\dagger f}{ }_{\vec{k}, s, n} \mathcal{V}^{f}(\vec{k}, s, n) e^{-i \vec{k} \cdot \vec{x}-i E_{n}^{f(-)} t}\right]
\end{aligned}
$$

where $c_{i}$ are the color matrices. For electrons:

$$
\begin{aligned}
\psi^{e}= & \frac{1}{\sqrt{V}} \sum_{\vec{k}, s, n}\left[A_{\vec{k}, s, n}^{e} \mathcal{U}^{e}(\vec{k}, s, n) e^{i \vec{k} \cdot \vec{x}-i E_{n}^{e(+)} t}\right. \\
& \left.+B_{\vec{k}, s, n}^{\dagger e} \mathcal{V}^{e}(\vec{k}, s, n) e^{-i \vec{k} \cdot \vec{x}-i E_{n}^{e(-)} t}\right]
\end{aligned}
$$

where the spinors satisfy:

$$
\mathcal{U}^{\dagger^{i}}\left(\vec{k}^{\prime}, s^{\prime}, n^{\prime}\right) \mathcal{U}^{i}(\vec{k}, s, n)=\mathcal{V}^{\dagger^{i}}\left(\vec{k}^{\prime}, s^{\prime}, n^{\prime}\right) \mathcal{V}^{i}(\vec{k}, s, n)=\delta_{\vec{k}^{\prime} \vec{k}} \delta_{s^{\prime} s} \delta_{n^{\prime} n}
$$

and

$$
\mathcal{U}^{\dagger^{i}}\left(\vec{k}^{\prime}, s^{\prime}, n^{\prime}\right) \mathcal{V}^{i}(\vec{k}, s, n)=\mathcal{V}^{\dagger^{i}}\left(\vec{k}^{\prime}, s^{\prime}, n^{\prime}\right) \mathcal{U}^{i}(\vec{k}, s, n)=0
$$

The creation annihilation operators:

$$
\left\{A^{i}, A^{\dagger^{i}}\right\}=\left\{B^{i}, B^{\dagger^{i}}\right\}=1
$$

and also

$$
\left\{A^{i}, B^{i}\right\}=\left\{A^{\dagger^{i}}, B^{\dagger^{i}}\right\}=0
$$

From (B.13) to (B.16) we have $i=e$ and $i=f=u, d, s$.

Substituting (B.12) and (B.14) in (B.8) we get:

$$
\hat{\mathcal{N}}_{e}=\frac{1}{V} \int d^{3} x \sum_{\vec{k}^{\prime}, s^{\prime}, n^{\prime}} \sum_{\vec{k}, s, n}\left\{A_{\vec{k}^{\prime}, s^{\prime}, n^{\prime}}^{\dagger e} \mathcal{U}^{\dagger e}\left(\vec{k}^{\prime}, s^{\prime}, n^{\prime}\right) A_{\vec{k}, s, n}^{e} \mathcal{U}^{e}(\vec{k}, s, n) e^{-i\left(\vec{k}^{\prime}-\vec{k}\right) \cdot \vec{x}} \times\right.
$$




$$
\left.e^{i\left(E_{n^{\prime}}^{e(+)}-E_{n}^{e(+)}\right) t}+B_{\vec{k}^{\prime}, s^{\prime}, n^{\prime}}^{e} \mathcal{V}^{\dagger e}\left(\vec{k}^{\prime}, s^{\prime}, n^{\prime}\right) B_{\vec{k}, s, n}^{\dagger e} \mathcal{V}^{e}(\vec{k}, s, n) e^{i\left(\vec{k}^{\prime}-\vec{k}\right) \cdot \vec{x}} e^{i\left(E_{n^{\prime}}^{e(-)}-E_{n}^{e(-)}\right) t}\right\}
$$

and using (B.13) we find:

$$
\hat{\mathcal{N}}_{e}=\frac{1}{V} \int d^{3} x \sum_{\vec{k}, s, n}\left\{A_{\vec{k}, s, n}^{\dagger e} A_{\vec{k}, s, n}^{e}+B_{\vec{k}, s, n}^{e} B_{\vec{k}, s, n}^{\dagger e}\right\}
$$

Applying normal ordering to the operators of antiparticles, i.e., using (B.15) in (B.17):

$$
\hat{\mathcal{N}}_{e}=\frac{1}{V} \int d^{3} x \sum_{\vec{k}, s, n}\left\{A_{\vec{k}, s, n}^{\dagger e} A_{\vec{k}, s, n}^{e}-B_{\vec{k}, s, n}^{\dagger e} B_{\vec{k}, s, n}^{e}+1\right\}
$$

Calculating (B.5) using (B.18) we have:

$$
\begin{gathered}
\hat{N}_{e}=\frac{1}{V} \int d^{3} x \sum_{\vec{k}, s, n}\left\{A_{\vec{k}, s, n}^{\dagger e} A_{\vec{k}, s, n}^{e}-B_{\vec{k}, s, n}^{\dagger e} B_{\vec{k}, s, n}^{e}\right\}+\frac{1}{V} \int d^{3} x \sum_{\vec{k}, s, n} 1 \\
-\left\langle 0\left|\frac{1}{V} \int d^{3} x \sum_{\vec{k}, s, n} A_{\vec{k}, s, n}^{\dagger e} A_{\vec{k}, s, n}^{e}\right| 0\right\rangle+\left\langle 0\left|\frac{1}{V} \int d^{3} x \sum_{\vec{k}, s, n} B_{\vec{k}, s, n}^{\dagger e} B_{\vec{k}, s, n}^{e}\right| 0\right\rangle \\
-\left\langle 0\left|\frac{1}{V} \int d^{3} x \sum_{\vec{k}, s, n} 1\right| 0\right\rangle
\end{gathered}
$$

resulting in:

$$
\hat{N}_{e}=\frac{1}{V} \int d^{3} x \sum_{\vec{k}, s, n}\left\{A_{\vec{k}, s, n}^{\dagger e} A_{\vec{k}, s, n}^{e}-B_{\vec{k}, s, n}^{\dagger e} B_{\vec{k}, s, n}^{e}\right\}
$$

Analogously for the quarks, effecting the sum in $i$ and $j$ :

$$
c_{i}^{\dagger} \delta_{i j} c_{j}=c_{1}^{\dagger} c_{1}+c_{2}^{\dagger} c_{2}+c_{3}^{\dagger} c_{3}=3
$$

But here we will make this as an average and return the color contributions in the degeneracy factor in the end, its equivalent.

$$
c_{i}^{\dagger} \delta_{i j} c_{j} \rightarrow \frac{c_{i}^{\dagger} \delta_{i j} c_{j}}{\text { (number of quark colors) }}=\frac{c_{1}^{\dagger} c_{1}+c_{2}^{\dagger} c_{2}+c_{3}^{\dagger} c_{3}}{3}=1
$$


So we found (B.6) in the form:

$$
\hat{N}_{f}=\frac{1}{V} \int d^{3} x \sum_{\vec{k}, s, n}\left\{A_{\vec{k}, s, n}^{\dagger f} A_{\vec{k}, s, n}^{f}-B_{\vec{k}, s, n}^{\dagger f} B_{\vec{k}, s, n}^{f}\right\}
$$

Before calculating the Hamiltonian from (B.7) and (B.10), we need some results. Doing $\bar{\psi}^{f} \times(2.32)$ with (B.11) and (B.14) we get:

$$
\begin{gathered}
\frac{1}{V} \sum_{\vec{k}, s, n, \vec{k}^{\prime}, s^{\prime}, n^{\prime}}\left\{A_{\vec{k}^{\prime}, s^{\prime}, n^{\prime}}^{\dagger f} \mathcal{U}^{\dagger f}\left(\vec{k}^{\prime}, s^{\prime}, n^{\prime}\right) e^{-i \vec{k}^{\prime} \cdot \vec{x}+i E_{n^{\prime}}^{f(+)} t}\right. \\
\left.+B_{\vec{k}^{\prime}, s^{\prime}, n^{\prime}}^{f} \mathcal{V}^{\dagger f}\left(\vec{k}^{\prime}, s^{\prime}, n^{\prime}\right) e^{i \vec{k}^{\prime} \cdot \vec{x}+i E_{n^{\prime}}^{f(-)} t}\right\} c_{i}^{\dagger}\left[i \delta_{i j} \partial_{0}+i \delta_{i j} \vec{\alpha} \cdot \vec{\nabla}+g_{h} T_{i j}^{a} \alpha_{0}^{a}\right. \\
\left.-\delta_{i j} \gamma^{0}\left(m_{f}+Q_{f} \gamma^{\mu} A_{\mu}\right)\right] c_{j}\left\{A_{\vec{k}, s, n}^{f} \mathcal{U}^{f}(\vec{k}, s, n) e^{i \vec{k} \cdot \vec{x}-i E_{n}^{f(+)} t}\right. \\
\left.+B^{\dagger f}{ }_{\vec{k}, s, n} \mathcal{V}^{f}(\vec{k}, s, n) e^{-i \vec{k} \cdot \vec{x}-i E_{n}^{f(-)} t}\right\}=0
\end{gathered}
$$

therefore:

$$
\begin{gathered}
\frac{1}{V} \sum_{\vec{k}, s, n, \vec{k}^{\prime}, s^{\prime}, n^{\prime}} c_{i}^{\dagger}\left[i \delta_{i j}\left(-i E_{n}^{f(+)}\right)+i \delta_{i j} \vec{\alpha} \cdot(i \vec{k})+g_{h} T_{i j}^{a} \alpha_{0}^{a}-\delta_{i j} \gamma^{0}\left(m_{f}+Q_{f} \gamma^{\mu} A_{\mu}\right)\right] c_{j} \\
\left\{A_{\vec{k}^{\prime}, s^{\prime}, n^{\prime}}^{\dagger f} \mathcal{U}^{\dagger f}\left(\vec{k}^{\prime}, s^{\prime}, n^{\prime}\right) A_{\vec{k}, s, n}^{f} \mathcal{U}^{f}(\vec{k}, s, n) e^{-i\left(\vec{k}^{\prime}-\vec{k}\right) \cdot \vec{x}+i\left[E_{n^{\prime}}^{f(+)}-E_{n}^{f(+)}\right] t}\right\} \\
+\frac{1}{V} \sum_{\vec{k}, s, n, \vec{k}^{\prime}, s^{\prime}, n^{\prime}} c_{i}^{\dagger}\left[i \delta_{i j}\left(-i E_{n}^{f(-)}\right)+i \delta_{i j} \vec{\alpha} \cdot(-i \vec{k})+g_{h} T_{i j}^{a} \alpha_{0}^{a}-\delta_{i j} \gamma^{0}\left(m_{f}+Q_{f} \gamma^{\mu} A_{\mu}\right)\right] c_{j} \\
\left\{B_{\vec{k}^{\prime}, s^{\prime}, n^{\prime}}^{f} \mathcal{V}^{\dagger f}\left(\vec{k}^{\prime}, s^{\prime}, n^{\prime}\right) B_{\vec{k}, s, n}^{\dagger f} \mathcal{V}^{f}(\vec{k}, s, n) e^{i\left(\vec{k}^{\prime}-\vec{k}\right) \cdot \vec{x}+i\left[E_{n^{\prime}}^{f(-)}-E_{n}^{f(-)}\right] t}\right\}=0
\end{gathered}
$$

and using (B.13) and (B.22) we find:

$$
\begin{array}{r}
\frac{1}{V} \sum_{\vec{k}, s, n}\left[E_{n}^{f(+)}-\vec{\alpha} \cdot \vec{k}+g_{h} \mathcal{A}-\gamma^{0}\left(m_{f}+Q_{f} \gamma^{\mu} A_{\mu}\right)\right] A_{\vec{k}, s, n}^{\dagger f} A_{\vec{k}, s, n}^{f} \\
+\frac{1}{V} \sum_{\vec{k}, s, n}\left[E_{n}^{f(-)}+\vec{\alpha} \cdot \vec{k}+g_{h} \mathcal{A}-\gamma^{0}\left(m_{f}+Q_{f} \gamma^{\mu} A_{\mu}\right)\right] B_{\vec{k}, s, n}^{f} B_{\vec{k}, s, n}^{\dagger f}=0
\end{array}
$$

since $c_{i}^{\dagger} T_{i j}^{a} c_{j} \alpha_{0}^{a}=\mathcal{A}$ 
From the expression (B.24) we get the energies:

$$
E_{n}^{f(+)}+g_{h} \mathcal{A}=\vec{\alpha} \cdot \vec{k}+\gamma^{0}\left(m_{f}+Q_{f} \gamma^{\mu} A_{\mu}\right)
$$

and

$$
E_{n}^{f(-)}+g_{h} \mathcal{A}=-\vec{\alpha} \cdot \vec{k}+\gamma^{0}\left(m_{f}+Q_{f} \gamma^{\mu} A_{\mu}\right)
$$

Using (2.42) in (2.45) we find:

$$
\tilde{E}_{n}^{f( \pm)} \equiv E_{n}^{f( \pm)}+g_{h} \mathcal{A}= \pm \sqrt{m_{f}^{2}+k_{z}^{2}+2 n\left|Q_{f}\right| B}
$$

Comparing (B.25) and (B.26) with (B.27):

$$
\begin{aligned}
& \vec{\alpha} \cdot \vec{k}+\gamma^{0}\left(m_{f}+Q_{f} \gamma^{\mu} A_{\mu}\right) \longrightarrow \sqrt{m_{f}^{2}+k_{z}^{2}+2 n\left|Q_{f}\right| B} \\
& \vec{\alpha} \cdot \vec{k}-\gamma^{0}\left(m_{f}+Q_{f} \gamma^{\mu} A_{\mu}\right) \longrightarrow \sqrt{m_{f}^{2}+k_{z}^{2}+2 n\left|Q_{f}\right| B}
\end{aligned}
$$

And the same goes for the electrons, without the average on colour factors and interaction with gluon terms. This is:

$$
\begin{aligned}
& \vec{\alpha} \cdot \vec{k}+\gamma^{0}\left(m_{e}+Q_{e} \gamma^{\mu} A_{\mu}\right) \longrightarrow \sqrt{m_{e}^{2}+k_{z}^{2}+2 n\left|Q_{e}\right| B} \\
& \vec{\alpha} \cdot \vec{k}-\gamma^{0}\left(m_{e}+Q_{e} \gamma^{\mu} A_{\mu}\right) \longrightarrow \sqrt{m_{e}^{2}+k_{z}^{2}+2 n\left|Q_{e}\right| B}
\end{aligned}
$$

Proceeding with (B.10), we have:

$$
\begin{gathered}
\hat{\mathcal{H}}=\int d^{3} x\left\{\sum_{f=u}^{d, s} i \psi_{i}^{\dagger} \delta_{i j}\left(\partial_{0} \psi_{j}^{f}\right)+i \psi_{i}^{\dagger} \delta_{i j}\left(\partial_{0} \psi_{j}^{e}\right)\right\} \\
+\int d^{3} x\left\{-\frac{m_{G}^{2}}{2} \alpha_{0}^{a} \alpha_{0}^{a}+b \phi_{0}^{4}+\frac{B^{2}}{8 \pi}\right\} \\
+\int d^{3} x\left\{-\bar{\psi}_{i}^{e}\left[i \gamma^{\mu}\left(\delta_{i j} \partial_{\mu}+i \delta_{i j} Q_{e} A_{\mu}\right)-\delta_{i j} m_{e}\right] \psi_{j}^{e}\right\} \\
+\int d^{3} x\left\{-\sum_{f=u}^{d, s} \bar{\psi}_{i}^{f}\left\{i \gamma^{\mu}\left[\delta_{i j} \partial_{\mu}+i \delta_{i j} Q_{f} A_{\mu}\right]+g_{h} \gamma^{0} T_{i j}^{a} \alpha_{0}^{a}-\delta_{i j} m_{f}\right\} \psi_{j}^{f}\right\}
\end{gathered}
$$


Note that naturally the temporal derivative terms of the fermions are cancelled and (B.32) is now:

$$
\begin{gathered}
\hat{\mathcal{H}}=\int d^{3} x\left\{-\frac{m_{G}^{2}}{2} \alpha_{0}^{a} \alpha_{0}^{a}+b \phi_{0}^{4}+\frac{B^{2}}{8 \pi}\right\} \\
+\int d^{3} x\left\{-\psi_{i}^{\dagger^{e}}\left[i \delta_{i j}(\vec{\alpha} \cdot \vec{\nabla})-\gamma^{0} \delta_{i j}\left(m_{e}+Q_{e} \gamma^{\mu} A_{\mu}\right)\right] \psi_{j}^{e}\right\} \\
+\int d^{3} x\left\{-\sum_{f=u}^{d, s} \psi_{i}^{\dagger}\left[i \delta_{i j}(\vec{\alpha} \cdot \vec{\nabla})-\gamma^{0} \delta_{i j}\left(m_{f}+Q_{f} \gamma^{\mu} A_{\mu}\right)\right] \psi_{j}^{f}-\sum_{f=u}^{d, s} g_{h} \psi_{i}^{\dagger f} T_{i j}^{a} \alpha_{0}^{a} \psi_{j}^{f}\right\}
\end{gathered}
$$

Substituting (B.11), (B.12), (B.22) and the results (B.28) to (B.31) in (B.33), we find:

$$
\begin{gathered}
\hat{\mathcal{H}}=\int d^{3} x\left\{-\frac{m_{G}^{2}}{2} \alpha_{0}^{a} \alpha_{0}^{a}+b \phi_{0}{ }^{4}+\frac{B^{2}}{8 \pi}\right\} \\
+\int d^{3} x\left\{\frac{1}{V} \sum_{\vec{k}, s, n} \sqrt{m_{e}^{2}+k_{z}^{2}+2 n\left|Q_{e}\right| B}\left[A_{\vec{k}, s, n}^{\dagger e} A_{\vec{k}, s, n}^{e}-B_{\vec{k}, s, n}^{e} B_{\vec{k}, s, n}^{\dagger e}\right]\right\} \\
+\int d^{3} x\left\{\frac{1}{V} \sum_{f=u}^{d, s} \sum_{\vec{k}, s, n} \sqrt{m_{f}^{2}+k_{z}^{2}+2 n\left|Q_{f}\right| B}\left[A_{\vec{k}, s, n}^{\dagger f} A_{\vec{k}, s, n}^{f}-B_{\vec{k}, s, n}^{f} B_{\vec{k}, s, n}^{\dagger f}\right]\right\} \\
-\int d^{3} x\left\{\frac{1}{V} \sum_{f=u}^{d, s} \sum_{\vec{k}, s, n} g_{h}\left(c_{i}^{\dagger} T_{i j}^{a} c_{j}\right) \alpha_{0}^{a}\left[A_{\vec{k}, s, n}^{\dagger f} A_{\vec{k}, s, n}^{f}+B_{\vec{k}, s, n}^{f} B_{\vec{k}, s, n}^{\dagger f}\right]\right\}
\end{gathered}
$$

Again, normal ordering of (B.15) we get:

$$
\begin{gathered}
\hat{\mathcal{H}}=\int d^{3} x\left\{-\frac{m_{G}^{2}}{2} \alpha_{0}^{a} \alpha_{0}^{a}+b \phi_{0}^{4}+\frac{B^{2}}{8 \pi}\right\} \\
+\int d^{3} x\left\{\frac{1}{V} \sum_{\vec{k}, s, n} \sqrt{m_{e}^{2}+k_{z}^{2}+2 n\left|Q_{e}\right| B}\left[A_{\vec{k}, s, n}^{\dagger e} A_{\vec{k}, s, n}^{e}+B_{\vec{k}, s, n}^{\dagger e} B_{\vec{k}, s, n}^{e}\right]\right\} \\
-\int d^{3} x \frac{1}{V} \sum_{\vec{k}, s, n}\left(\sqrt{m_{e}^{2}+k_{z}^{2}+2 n\left|Q_{e}\right| B}\right) 1 \\
+\int d^{3} x\left\{\frac{1}{V} \sum_{f=u}^{d, s} \sum_{\vec{k}, s, n} \sqrt{m_{f}^{2}+k_{z}^{2}+2 n\left|Q_{f}\right| B}\left[A_{\vec{k}, s, n}^{\dagger f} A_{\vec{k}, s, n}^{f}+B_{\vec{k}, s, n}^{\dagger f} B_{\vec{k}, s, n}^{f}\right]\right\} \\
-\int d^{3} x \frac{1}{V} \sum_{f=u}^{d, s} \sum_{\vec{k}, s, n}\left(\sqrt{m_{f}^{2}+k_{z}^{2}+2 n\left|Q_{f}\right| B}\right) 1
\end{gathered}
$$




$$
\begin{gathered}
-\int d^{3} x\left\{\frac{1}{V} \sum_{f=u}^{d, s} \sum_{\vec{k}, s, n} g_{h}\left(c_{i}^{\dagger} T_{i j}^{a} c_{j}\right) \alpha_{0}^{a}\left[A_{\vec{k}, s, n}^{\dagger f} A_{\vec{k}, s, n}^{f}-B_{\vec{k}, s, n}^{\dagger f} B_{\vec{k}, s, n}^{f}\right]\right\} \\
-\int d^{3} x \frac{1}{V} \sum_{f=u}^{d, s} \sum_{\vec{k}, s, n}\left[g_{h}\left(c_{i}^{\dagger} T_{i j}^{a} c_{j}\right) \alpha_{0}^{a}\right] 1
\end{gathered}
$$

which is the operator described in (B.10). Now we can find the Hamiltonian (B.7) using (B.35):

$$
\begin{aligned}
& \hat{H}=\hat{\mathcal{H}}-\langle 0|\hat{\mathcal{H}}| 0\rangle=\int d^{3} x\left\{-\frac{m_{G}{ }^{2}}{2} \alpha_{0}^{a} \alpha_{0}^{a}+b \phi_{0}{ }^{4}+\frac{B^{2}}{8 \pi}\right\} \\
& +\int d^{3} x\left\{\frac{1}{V} \sum_{\vec{k}, s, n} \sqrt{m_{e}^{2}+k_{z}^{2}+2 n\left|Q_{e}\right| B}\left[A_{\vec{k}, s, n}^{\dagger e} A_{\vec{k}, s, n}^{e}+B_{\vec{k}, s, n}^{\dagger e} B_{\vec{k}, s, n}^{e}\right]\right\} \\
& -\int d^{3} x \frac{1}{V} \sum_{\vec{k}, s, n}\left(\sqrt{m_{e}^{2}+k_{z}^{2}+2 n\left|Q_{e}\right| B}\right) 1 \\
& +\int d^{3} x\left\{\frac{1}{V} \sum_{f=u}^{d, s} \sum_{\vec{k}, s, n} \sqrt{m_{f}^{2}+k_{z}^{2}+2 n\left|Q_{f}\right| B}\left[A_{\vec{k}, s, n}^{\dagger f} A_{\vec{k}, s, n}^{f}+B_{\vec{k}, s, n}^{\dagger f} B_{\vec{k}, s, n}^{f}\right]\right\} \\
& -\int d^{3} x \frac{1}{V} \sum_{f=u}^{d, s} \sum_{\vec{k}, s, n}\left(\sqrt{m_{f}^{2}+k_{z}^{2}+2 n\left|Q_{f}\right| B}\right) 1 \\
& -\int d^{3} x\left\{\frac{1}{V} \sum_{f=u}^{d, s} \sum_{\vec{k}, s, n} g_{h}\left(c_{i}^{\dagger} T_{i j}^{a} c_{j}\right) \alpha_{0}^{a}\left[A_{\vec{k}, s, n}^{\dagger f} A_{\vec{k}, s, n}^{f}-B_{\vec{k}, s, n}^{\dagger f} B_{\vec{k}, s, n}^{f}\right]\right\} \\
& -\int d^{3} x \frac{1}{V} \sum_{f=u}^{d, s} \sum_{\vec{k}, s, n}\left[g_{h}\left(c_{i}^{\dagger} T_{i j}^{a} c_{j}\right) \alpha_{0}^{a}\right] 1 \\
& -\left\langle 0\left|\int d^{3} x\left\{\frac{1}{V} \sum_{\vec{k}, s, n} \sqrt{m_{e}^{2}+k_{z}^{2}+2 n\left|Q_{e}\right| B}\left[A_{\vec{k}, s, n}^{\dagger e} A_{\vec{k}, s, n}^{e}+B_{\vec{k}, s, n}^{\dagger e} B_{\vec{k}, s, n}^{e}\right]\right\}\right| 0\right\rangle \\
& +\left\langle 0\left|\int d^{3} x \frac{1}{V} \sum_{\vec{k}, s, n}\left(\sqrt{m_{e}^{2}+k_{z}^{2}+2 n\left|Q_{e}\right| B}\right) 1\right| 0\right\rangle \\
& -\left\langle 0\left|\int d^{3} x\left\{\frac{1}{V} \sum_{f=u}^{d, s} \sum_{\vec{k}, s, n} \sqrt{m_{f}^{2}+k_{z}^{2}+2 n\left|Q_{f}\right| B}\left[A_{\vec{k}, s, n}^{\dagger f} A_{\vec{k}, s, n}^{f}+B_{\vec{k}, s, n}^{\dagger f} B_{\vec{k}, s, n}^{f}\right]\right\}\right| 0\right\rangle \\
& +\left\langle 0\left|\int d^{3} x \frac{1}{V} \sum_{f=u}^{d, s} \sum_{\overrightarrow{k, s, n}}\left(\sqrt{m_{f}^{2}+k_{z}^{2}+2 n\left|Q_{f}\right| B}\right) 1\right| 0\right\rangle
\end{aligned}
$$




$$
\begin{gathered}
+\left\langle 0\left|\int d^{3} x\left\{\frac{1}{V} \sum_{f=u}^{d, s} \sum_{\vec{k}, s, n} g_{h}\left(c_{i}^{\dagger} T_{i j}^{a} c_{j}\right) \alpha_{0}^{a}\left[A_{\vec{k}, s, n}^{\dagger f} A_{\vec{k}, s, n}^{f}-B_{\vec{k}, s, n}^{\dagger f} B_{\vec{k}, s, n}^{f}\right]\right\}\right| 0\right\rangle \\
+\left\langle 0\left|\int d^{3} x \frac{1}{V} \sum_{f=u}^{d, s} \sum_{\vec{k}, s, n}\left[g_{h}\left(c_{i}^{\dagger} T_{i j}^{a} c_{j}\right) \alpha_{0}^{a}\right] 1\right| 0\right\rangle
\end{gathered}
$$

Which results in:

$$
\begin{gathered}
\hat{H}=\int d^{3} x\left\{-\frac{m_{G}^{2}}{2} \alpha_{0}^{a} \alpha_{0}^{a}+b \phi_{0}^{4}+\frac{B^{2}}{8 \pi}\right\} \\
+\int d^{3} x\left\{\frac{1}{V} \sum_{\vec{k}, s, n} \sqrt{m_{e}^{2}+k_{z}^{2}+2 n\left|Q_{e}\right| B}\left[A_{\vec{k}, s, n}^{\dagger e} A_{\vec{k}, s, n}^{e}+B_{\vec{k}, s, n}^{\dagger e} B_{\vec{k}, s, n}^{e}\right]\right\} \\
+\int d^{3} x\left\{\frac{1}{V} \sum_{f=u}^{d, s} \sum_{\vec{k}, s, n} \sqrt{m_{f}^{2}+k_{z}^{2}+2 n\left|Q_{f}\right| B}\left[A_{\vec{k}, s, n}^{\dagger f} A_{\vec{k}, s, n}^{f}+B_{\vec{k}, s, n}^{\dagger f} B_{\vec{k}, s, n}^{f}\right]\right\} \\
-\int d^{3} x\left\{\frac{1}{V} \sum_{f=u}^{d, s} \sum_{\vec{k}, s, n} g_{h}\left(c_{i}^{\dagger} T_{i j}^{a} c_{j}\right) \alpha_{0}^{a}\left[A_{\vec{k}, s, n}^{\dagger f} A_{\vec{k}, s, n}^{f}-B_{\vec{k}, s, n}^{\dagger f} B_{\vec{k}, s, n}^{f}\right]\right\}
\end{gathered}
$$

Substituting (B.23) in the last term of (B.36) we arrive at:

$$
\begin{gathered}
\hat{H}=\int d^{3} x\left\{-\frac{m_{G}^{2}}{2} \alpha_{0}^{a} \alpha_{0}^{a}+b \phi_{0}^{4}+\frac{B^{2}}{8 \pi}\right\} \\
+\int d^{3} x\left\{\frac{1}{V} \sum_{\vec{k}, s, n} \sqrt{m_{e}^{2}+k_{z}^{2}+2 n\left|Q_{e}\right| B}\left[A_{\vec{k}, s, n}^{\dagger e} A_{\vec{k}, s, n}^{e}+B_{\vec{k}, s, n}^{\dagger e} B_{\vec{k}, s, n}^{e}\right]\right\} \\
+\int d^{3} x\left\{\frac{3}{V} \sum_{f=u}^{d, s} \sum_{\vec{k}, s, n} \sqrt{m_{f}^{2}+k_{z}^{2}+2 n\left|Q_{f}\right| B}\left[A_{\vec{k}, s, n}^{\dagger f} A_{\vec{k}, s, n}^{f}+B_{\vec{k}, s, n}^{\dagger f} B_{\vec{k}, s, n}^{f}\right]\right\} \\
-\sum_{f=u}^{d, s} g_{h}\left(c_{i}^{\dagger} T_{i j}^{a} c_{j}\right) \alpha_{0}^{a} \hat{N}_{f}
\end{gathered}
$$

Now returning to the calculus of the partition function. Inserting (B.20), (B.23) and (B.37) in (B.2) we get:

$$
\begin{gathered}
Z=\operatorname{Tr}\left\{\exp \left[-\left(\hat{H}-\mu_{e} \hat{N}_{e}-\sum_{f=u}^{d, s} \mu_{f} \hat{N}_{f}\right) / T\right]\right\} \\
=\exp \left[\int d^{3} x\left\{-\frac{m_{G}^{2}}{2} \alpha_{0}^{a} \alpha_{0}^{a}+b \phi_{0}^{4}+\frac{B^{2}}{8 \pi}\right\} \frac{1}{T}\right] \times
\end{gathered}
$$




$$
\begin{gathered}
\times \operatorname{Tr}\left\{\operatorname { e x p } \left\{\int d^{3} x\left[-\frac{1}{V T} \sum_{\vec{k}, s, n} \sqrt{m_{e}^{2}+k_{z}^{2}+2 n\left|Q_{e}\right| B}\left(A_{\vec{k}, s, n}^{\dagger e} A_{\vec{k}, s, n}^{e}+B_{\vec{k}, s, n}^{\dagger e} B_{\vec{k}, s, n}^{e}\right)\right]\right.\right. \\
+\int d^{3} x\left[-\frac{1}{V T} \sum_{f=u}^{d, s} \sum_{\vec{k}, s, n} \sqrt{m_{f}^{2}+k_{z}^{2}+2 n\left|Q_{f}\right| B}\left(A_{\vec{k}, s, n}^{\dagger f} A_{\vec{k}, s, n}^{f}+B_{\vec{k}, s, n}^{\dagger f} B_{\vec{k}, s, n}^{f}\right)\right] \\
+\sum_{f=u}^{d, s} \frac{g_{h}}{T}\left(c_{i}^{\dagger} T_{i j}^{a} c_{j}\right) \alpha_{0}^{a} \frac{1}{V} \int d^{3} x \sum_{\vec{k}, s, n}\left(A_{\vec{k}, s, n}^{\dagger f} A_{\vec{k}, s, n}^{f}-B_{\vec{k}, s, n}^{\dagger f} B_{\vec{k}, s, n}^{f}\right) \\
+\frac{1}{T} \mu_{e} \frac{1}{V} \int d^{3} x \sum_{\vec{k}, s, n}\left(A_{\vec{k}, s, n}^{\dagger e} A_{\vec{k}, s, n}^{e}-B_{\vec{k}, s, n}^{\dagger e} B_{\vec{k}, s, n}^{e}\right) \\
\left.\left.+\frac{1}{T} \sum_{f=u}^{d, s} \mu_{f} \frac{1}{V} \int d^{3} x \sum_{\vec{k}, s, n}\left(A_{\vec{k}, s, n}^{\dagger f} A_{\vec{k}, s, n}^{f}-B_{\vec{k}, s, n}^{\dagger f} B_{\vec{k}, s, n}^{f}\right)\right\}\right\}
\end{gathered}
$$

and therefore:

$$
\begin{gathered}
Z=\exp \left[\int d^{3} x\left\{-\frac{m_{G}^{2}}{2} \alpha_{0}^{a} \alpha_{0}^{a}+b \phi_{0}^{4}+\frac{B^{2}}{8 \pi}\right\} \frac{1}{T}\right] \times \\
\times \sum_{n_{1}, n_{2}, \ldots, n_{\infty}}\left\langle n_{1}, n_{2}, \ldots, n_{\infty}\right| \exp \left\{\int d ^ { 3 } x \left[-\frac{1}{V T} \sum_{\vec{k}, s, n} \sqrt{m_{e}^{2}+k_{z}^{2}+2 n\left|Q_{e}\right| B} \times\right.\right. \\
\left.\times\left(A_{\vec{k}, s, n}^{\dagger e} A_{\vec{k}, s, n}^{e}+B_{\vec{k}, s, n}^{\dagger e} B_{\vec{k}, s, n}^{e}\right)\right] \\
+\int d^{3} x\left[-\frac{1}{V T} \sum_{f=u}^{d, s} \sum_{\vec{k}, s, n} \sqrt{m_{f}^{2}+k_{z}^{2}+2 n\left|Q_{f}\right| B}\left(A_{\vec{k}, s, n}^{\dagger f} A_{\vec{k}, s, n}^{f}+B_{\vec{k}, s, n}^{\dagger f} B_{\vec{k}, s, n}^{f}\right)\right] \\
+\sum_{f=u}^{d, s} \frac{g_{h}}{T}\left(c_{i}^{\dagger} T_{i j}^{a} c_{j}\right) \alpha_{0}^{a} \frac{1}{V} \int d^{3} x \sum_{\vec{k}, s, n}\left(A_{\vec{k}, s, n}^{\dagger f} A_{\vec{k}, s, n}^{f}-B_{\vec{k}, s, n}^{\dagger f} B_{\vec{k}, s, n}^{f}\right) \\
+\frac{1}{T} \mu_{e} \frac{1}{V} \int d^{3} x \sum_{\vec{k}, s, n}\left(A_{\vec{k}, s, n}^{\dagger e} A_{\vec{k}, s, n}^{e}-B_{\vec{k}, s, n}^{\dagger e} B_{\vec{k}, s, n}^{e}\right) \\
\left.+\frac{1}{T} \sum_{f=u}^{d, s} \mu_{f} \frac{1}{V} \int d^{3} x \sum_{\vec{k}, s, n}\left(A_{\vec{k}, s, n}^{\dagger f} A_{\vec{k}, s, n}^{f}-B_{\vec{k}, s, n}^{\dagger f} B_{\vec{k}, s, n}^{f}\right)\right\}\left|n_{1}, n_{2}, \ldots, n_{\infty}\right\rangle \quad(\mathrm{B} .38)
\end{gathered}
$$

Following the steps in (B.3) we have (B.38):

$$
Z=\exp \left[V\left\{-\frac{m_{G}{ }^{2}}{2} \alpha_{0}^{a} \alpha_{0}^{a}+b \phi_{0}{ }^{4}+\frac{B^{2}}{8 \pi}\right\} \frac{1}{T}\right] \times
$$




$$
\begin{aligned}
& \times \sum_{n_{1}}\left\langle n_{1}\right| \exp \left\{\left[-\frac{1}{T} \sqrt{m_{e}^{2}+k_{z}^{2}+2 n\left|Q_{e}\right| B}\right] n_{1}-\left[\frac{1}{T} \sum_{f=u}^{d, s} \sqrt{m_{f}^{2}+k_{z}^{2}+2 n\left|Q_{f}\right| B}\right] n_{1}\right. \\
& +\left[\sum_{f=u}^{d, s} \frac{g_{h}}{T}\left(c_{i}^{\dagger} T_{i j}^{a} c_{j}\right) \alpha_{0}^{a}+\frac{\mu_{e}}{T}+\frac{1}{T} \sum_{f=u}^{d, s} \mu_{f}\right] n_{1} \\
& -\left[\frac{1}{T} \sqrt{m_{e}^{2}+k_{z}^{2}+2 n\left|Q_{e}\right| B}\right] n_{1}^{*}-\left[\frac{1}{T} \sum_{f=u}^{d, s} \sqrt{m_{f}^{2}+k_{z}^{2}+2 n\left|Q_{f}\right| B}\right] n_{1}^{*} \\
& \left.-\left[\sum_{f=u}^{d, s} \frac{g_{h}}{T}\left(c_{i}^{\dagger} T_{i j}^{a} c_{j}\right) \alpha_{0}^{a}+\frac{\mu_{e}}{T}+\frac{1}{T} \sum_{f=u}^{d, s} \mu_{f}\right] n_{1}^{*}\right\}\left|n_{1}\right\rangle \times \\
& \times \sum_{n_{2}}\left\langle n_{2}\right| \exp \left\{\left[-\frac{1}{T} \sqrt{m_{e}^{2}+k_{z}^{2}+2 n\left|Q_{e}\right| B}\right] n_{2}\right. \\
& -\left[\frac{1}{T} \sum_{f=u}^{d, s} \sqrt{m_{f}^{2}+k_{z}^{2}+2 n\left|Q_{f}\right| B}\right] n_{2} \\
& +\left[\sum_{f=u}^{d, s} \frac{g_{h}}{T}\left(c_{i}^{\dagger} T_{i j}^{a} c_{j}\right) \alpha_{0}^{a}+\frac{\mu_{e}}{T}+\frac{1}{T} \sum_{f=u}^{d, s} \mu_{f}\right] n_{2} \\
& -\left[\frac{1}{T} \sqrt{m_{e}^{2}+k_{z}^{2}+2 n\left|Q_{e}\right| B}\right] n_{2}^{*}-\left[\frac{1}{T} \sum_{f=u}^{d, s} \sqrt{m_{f}^{2}+k_{z}^{2}+2 n\left|Q_{f}\right| B}\right] n_{2}^{*} \\
& \left.-\left[\sum_{f=u}^{d, s} \frac{g_{h}}{T}\left(c_{i}^{\dagger} T_{i j}^{a} c_{j}\right) \alpha_{0}^{a}+\frac{\mu_{e}}{T}+\frac{1}{T} \sum_{f=u}^{d, s} \mu_{f}\right] n_{2}^{*}\right\}\left|n_{2}\right\rangle \ldots \\
& \ldots \sum_{n_{\infty}}\left\langle n_{\infty}\right| \exp \left\{\left[-\frac{1}{T} \sqrt{m_{e}^{2}+k_{z}^{2}+2 n\left|Q_{e}\right| B}\right] n_{\infty}\right. \\
& -\left[\frac{1}{T} \sum_{f=u}^{d, s} \sqrt{m_{f}^{2}+k_{z}^{2}+2 n\left|Q_{f}\right| B}\right] n_{\infty} \\
& +\left[\sum_{f=u}^{d, s} \frac{g_{h}}{T}\left(c_{i}^{\dagger} T_{i j}^{a} c_{j}\right) \alpha_{0}^{a}+\frac{\mu_{e}}{T}+\frac{1}{T} \sum_{f=u}^{d, s} \mu_{f}\right] n_{\infty} \\
& -\left[\frac{1}{T} \sqrt{m_{e}^{2}+k_{z}^{2}+2 n\left|Q_{e}\right| B}\right] n_{\infty}^{*}-\left[\frac{1}{T} \sum_{f=u}^{d, s} \sqrt{m_{f}^{2}+k_{z}^{2}+2 n\left|Q_{f}\right| B}\right] n_{\infty}^{*} \\
& \left.-\left[\sum_{f=u}^{d, s} \frac{g_{h}}{T}\left(c_{i}^{\dagger} T_{i j}^{a} c_{j}\right) \alpha_{0}^{a}+\frac{\mu_{e}}{T}+\frac{1}{T} \sum_{f=u}^{d, s} \mu_{f}\right] n_{\infty}^{*}\right\}\left|n_{\infty}\right\rangle
\end{aligned}
$$

where $\sum_{n_{i}}=\sum_{\vec{k}, s, n}$ for the state $\left|n_{i}\right\rangle$ to $i=1,2, \ldots, \infty$. The number $n_{i}$ 
refers to the actuation of the operators $A_{\vec{k}, s, n}^{\dagger j} A_{\vec{k}, s, n}^{j}$ and $n_{i}^{*}$ to the operators $B_{\vec{k}, s, n}^{\dagger j} B_{\vec{k}, s, n}^{j}$ for fermions $j=e, u, d, s$.

Carrying on the calculations that result in (B.4) for the fermions we rewrite (B.39) as:

$$
\begin{gathered}
Z=\exp \left[V\left\{-\frac{m_{G}{ }^{2}}{2} \alpha_{0}^{a} \alpha_{0}^{a}+b \phi_{0}{ }^{4}+\frac{B^{2}}{8 \pi}\right\} \frac{1}{T}\right] \times \\
\times \prod_{\vec{k}, s, n} \sum_{n_{e}=0}^{1} \sum_{n_{e}{ }^{*}=0}^{1}\left\{\exp \left[-\left(\mathcal{E}_{n}^{e}-\mu_{e}\right) n_{e} / T\right] \exp \left[-\left(\mathcal{E}_{n}^{e}+\mu_{e}\right) n_{e}{ }^{*} / T\right]\right\} \\
\times \prod_{f=u}^{d, s} \prod_{\vec{k}, s, n} \sum_{n_{f}=0}^{1} \sum_{n_{f}^{*}=0}^{1}\left\{\exp \left[-\left(\mathcal{E}_{n}^{f}-\nu_{f}\right) n_{f} / T\right] \exp \left[-\left(\mathcal{E}_{n}^{f}+\nu_{f}\right) n_{f}{ }^{*} / T\right]\right\}
\end{gathered}
$$

or:

$$
\begin{gathered}
Z=\exp \left[V\left\{-\frac{m_{G}{ }^{2}}{2} \alpha_{0}^{a} \alpha_{0}^{a}+b \phi_{0}{ }^{4}+\frac{B^{2}}{8 \pi}\right\} \frac{1}{T}\right] \times \\
\times \prod_{\vec{k}, s, n}\left\{1+\exp \left[-\left(\mathcal{E}_{n}^{e}-\mu_{e}\right) / T\right]\right\}\left\{1+\exp \left[-\left(\mathcal{E}_{n}^{e}+\mu_{e}\right) / T\right]\right\} \\
\times \prod_{f=u}^{d, s} \prod_{\vec{k}, s, n}\left\{1+\exp \left[-\left(\mathcal{E}_{n}^{f}-\nu_{f}\right) / T\right]\right\}\left\{1+\exp \left[-\left(\mathcal{E}_{n}^{f}+\nu_{f}\right) / T\right]\right\}
\end{gathered}
$$

where the energies are:

$$
\begin{aligned}
& \mathcal{E}_{n}^{e}=\sqrt{m_{e}^{2}+k_{z}^{2}+2 n\left|Q_{e}\right| B} \\
& \mathcal{E}_{n}^{f}=\sqrt{m_{f}^{2}+k_{z}^{2}+2 n\left|Q_{f}\right| B}
\end{aligned}
$$

and the quark $f$ chemical potential is given by:

$$
\nu_{f} \equiv \mu_{f}+g_{h}\left(c_{i}^{\dagger} T_{i j}^{a} c_{j}\right) \alpha_{0}^{a}
$$




\section{Bibliography}

[1] D. A. Fogaça, S. M. Sanches, T. F. Motta, and F. S. Navarra. "Compact stars with strongly coupled quark matter in a strong magnetic field". In: Phys. Rev. C94.5 (2016), p. 055805. DOI: 10 . 1103 / PhysRevC . 94. 055805. arXiv: 1608.00602 [hep-ph].

[2] Taizo Muta. Foundations of Quantum Chromodynamics: An Introduction to Perturbative Methods in Gauge Theories, (3rd ed.) Vol. 78. World scientific Lecture Notes in Physics. Hackensack, N.J.: World Scientific, 2010. ISBN: 9789812793539. URL: http: / /www-spires . fnal .gov/ spires/find/books/www?cl=0C793.3.035M88::2010.

[3] Gert Aarts. "Introductory lectures on lattice QCD at nonzero baryon number". In: J. Phys. Conf. Ser. 706.2 (2016). DOI: 10 . $1088 / 1742-$ 6596/706/2/022004. arXiv: 1512.05145.

[4] Amâncio CS Friaça, Elisabete DAL PINO, Laerte Sodré Jr, and Vera JATENCO-PEREIRA. “Astronomia: uma visão geral do universo". In: São Paulo: EDUSP (2000).

[5] URL: http://hyperphysics.phy-astr.gsu.edu.

[6] Edward Farhi and R. L. Jaffe. "Strange Matter". In: Phys. Rev. D30 (1984), p. 2379. DOI: 10.1103/PhysRevD . 30.2379.

[7] N. K. Glendenning. Compact stars: Nuclear physics, particle physics, and general relativity. 1997.

[8] J. R. Oppenheimer and G. M. Volkoff. "On Massive Neutron Cores". In: Phys. Rev. 55 (4 1939), pp. 374-381. DOI: 10 . 1103 / PhysRev . 55. 374. URL: http://link.aps.org/doi/10.1103/PhysRev. 55. 374.

[9] Paul Demorest et al. "Shapiro Delay Measurement of A Two Solar Mass Neutron Star". In: Nature 467 (2010), pp. 1081-1083. DOI: 10 . 1038/nature0 9466. arXiv: 1010.5788 [astro-ph.HE]. 
[10] John Antoniadis et al. "A Massive Pulsar in a Compact Relativistic Binary". In: Science 340 (2013), p. 6131. DOI: 10 . 1126 / science . 1233232. arXiv: 1304.6875 [astro-ph.HE] .

[11] M. H. van Kerkwijk, R. Breton, and S. R. Kulkarni. In: Astrophys J. 728 95 (2011).

[12] Franzon, Bruno Cezar de Souza. "Gluons em estrelas de nêutrons". MA thesis. Universidade de São Paulo, 2012.

[13] James M. Lattimer and Madappa Prakash. "What a Two Solar Mass Neutron Star Really Means". In: 2011. arXiv: 1012.3208 [astro-ph.SR].

[14] Alice K. Harding. “The Neutron Star Zoo”. In: Front. Phys.(Beijing) 8 (2013), pp. 679-692. DOI: 10 . 1007 / s11467-013-0285-0. arXiv: 1302.0869 [astro-ph. HE].

[15] B. Franzon, D. A. Fogaca, F. S. Navarra, and J. E. Horvath. "Self-bound Interacting QCD Matter in Compact Stars". In: Phys. Rev. D86 (2012), p. 065031. DOI: 10.1103/PhysRevD . 86.065031 . arXiv: 1203. 6090 [astro-ph.SR].

[16] D. P. Menezes and L. L. Lopes. In: Eur. Phys. J. A 52, 17 (2016).

[17] L. L. Lopes and D. Menezes. In: JCAP 1508, 002 (2015).

[18] J. E. Horvath D. M. Paret and A. P. Martinez. In: ().

[19] L. Paulucci, E. J. Ferrer, V. de la Incera, and J. E. Horvath. In: Phys Rev. D 83, 043009 (2011).

[20] Bruno Franzon and Stefan Schramm. "Effects of strong magnetic fields and rotation on white dwarf structure". In: Phys. Rev. D92.8 (2015), p. 083006. DOI: 10.1103/PhysRevD.92.083006. arXiv: 1507 . 05557 [astro-ph.SR].

[21] M. Bocquet, S. Bonazzola, E. Gourgoulhon, and J. Novak. In: Astron . Astrophys. J. 301, 757 (1995).

[22] C. Y. Cardall, M. Prakash, And J. M. Lattimer. "Effects Of Strong Magnetic Fields On Neutron Star Structure". In: The Astroph. J., 554 : 322339 (2001). 
[23] D. A. Fogaca and F. S. Navarra. "Gluon condensates in a cold quark-gluon plasma". In: Phys. Lett. B700 (2011), pp. 236-242. DOI: 10 . 1016 / j . physletb.2011.05.011. arXiv: 1012.5266 [hep-ph] .

[24] L. S. Celenza and C. M. Shakin. "Description of the Gluon Condensate". In: Phys. Rev. D34 (1986), pp. 1591-1600. DOI: 10.1103 /Phy sRevd . 34.1591.

[25] Xiang-dong Li and C. M. Shakin. "Description of gluon propagation in the presence of an $A^{* * 2}$ condensate". In: Phys. Rev. D71 (2005), p. 074007. DOI: 10 . 1103 / PhysRevD . 71.074007 . arXiv: hepph/0410404 [hep-ph].

[26] B. D. Serot and J. D. Walecka. In: Advances in Nuclear Physics 16, 1 (1986).

[27] J. D. Walecka. Theoretical Nuclear and Subnuclear Physics. Second. Imperial College Press and World Scientific Publishing Co. Pte. Ltd, 2004.

[28] H. Tezuka. "Mean Field Approximation to QCD". In: INS-Rep 643 (1987).

[29] I. Lovas, W. Greiner, P. Hraskǿ, and E. Lovas. In: Phys. Lett. B 156, 255 (1985).

[30] D. A. Fogaca, F. S. Navarra, and L. G. Ferreira Filho. "KdV solitons in a cold quark gluon plasma". In: Phys. Rev. D84 (2011), p. 054011. DOI: 10.1103/PhysRevD .84.054011. arXiv: 1106.5959 [hep-ph] .

[31] D. A. Fogaça, L. G. Ferreira Filho, and F. S. Navarra. In: Nucl. Phys. A 819150 (2009).

[32] Kaushik Bhattacharya and Palash B. Pal. In: Pramana 62, 1041 (2004).

[33] M. Chaichian et al. "Quantum magnetic and gravitational collapse". In: Phys. Rev. Lett. 84 (2000), pp. 5261-5264. DOI: 10.1103 / Phy sRevLett. 84.5261. arXiv: hep-ph/9911218 [hep-ph] .

[34] R. Gonzalez Felipe, A. Perez Martinez, H. Perez Rojas, and M. Orsaria. "Magnetized strange quark matter and magnetized strange quark stars". In: Phys. Rev. C77 (2008), p. 015807. DOI: 10 . 1103 / PhysRevC . 77. 015807. arXiv: 0709.1224 [astro-ph]. 
[35] V. Dexheimer, D. P. Menezes, and M. Strickland. In: J. Phys G 41, 15203 (2014).

[36] M. Strickand, V. Dexheimer, and D. P. Menezes. In: Phys. Rev. D 86, 125032 (2012).

[37] S. Chakrabarty. In: Phys. Rev. D 52, 1306 (1996).

[38] D. Manreza Paret and A. Perez Martinez. In: ().

[39] C. J. Xia J. X. Hou G. X. Peng and J. F. Xu. In: ().

[40] R. J. Furnstahl and Brian D. Serot. In: Phys. Rev. C 26241 (1990).

[41] Rodrigo Alvares de Souza. "Existência de matéria de quarks fria na Natureza: Modelos e Observações". PhD thesis. 2016.

[42] Bruno Franzon, V. Dexheimer, and S. Schramm. "Effects of strong magnetic fields on hybrid stars". In: PoS MPCS2015 (2016), p. 018.

[43] B. Franzon, V. Dexheimer, and S. Schramm. "A self-consistent study of magnetic field effects on hybrid stars". In: Mon. Not. Roy. Astron. Soc. 456.3 (2016), pp. 2937-2945. DOI: 10 . 1093 / mnras / stv2606. arXiv: 1508.04431 [astro-ph.HE] . 